\title{
Sédimentologie, Granulométrie, Analyse Séquentielle et Paléoenvironnements du Remplissage Holocène Récent de la Baie de Loango dans le Secteur de Kivesso (République du Congo)
}

\section{Miyouna Timothée, Docteur Maître-Assistant}

Université Marien NGOUABI, Faculté des Sciences et Techniques, Laboratoire des Géosciences, République du Congo

Elenga Hilaire, Maître de Conférences

Centre de Recherches Géologiques et Minières (CRGM), Brazzaville, République du Congo

Essouli Olivier Florent, Docteur Maître-Assistant

Université Marien NGOUABI, Faculté des Sciences et Techniques, Laboratoire des Géosciences, Brazzaville, République du Congo

Koubetchi Tsimba Jeannalpha Dorlya, Université Marien NGOUABI, Faculté des Sciences et Techniques, Laboratoire des Géosciences, Brazzaville, République du Congo

Malounguila-Nganga Dieudonné Maurice, Centre de Recherches Géologiques et Minières (CRGM), Brazzaville, République du Congo

Makoundi Issouessou Queen Dietrich, Université Marien NGOUABI, Faculté des Sciences et Techniques, Laboratoire des Géosciences, Brazzaville, République du Congo Boudzoumou Florent, Maître de Conférences Institut de Recherche en Sciences Exactes et Naturelles (IRSEN), Brazzaville, République du Congo

\section{Résumé}

L'étude sédimentologique, granulométrique, minéralogique et séquentielle associée aux datations au radiocarbone $\left({ }^{14} \mathrm{C}\right)$ a été réalisée dans la baie de Loango dans le secteur de Kivesso. Elle a pour but de mieux comprendre la nature, l'origine, les mécanismes de transport, les paléoenvironnements de sédimentation ainsi que l'organisation séquentielle du remplissage sédimentaire Holocène récent de la baie de Loango. L'étude met en évidence une zone marécageuse qui s'est développée entre 500 ans B.P. et 300 ans B.P. sur un cordon sableux littoral, édifié lors de la dernière 
régression Holocène qui couvrait le secteur de Kivesso. Cette zone marécageuse peu profonde et couverte par une forêt ombrophile a été progressivement colmatée par des sédiments provenant de la série des Gorges de Diosso et transportés par des cours d'eau à compétence contrastée, parfois torrentielle. Le dépôt est structuré en séquences élémentaires fluviatiles méandriformes de type chenaux. Deux méga séquences correspondant à deux grandes phases de colmatage ont été mises en évidence. La première méga séquence datée entre 500 ans B.P. et 300 ans B.P. correspond aux unités 2 et 3. Elle marque le développement des marécages et de leur colmatage par les cours d'eaux côtiers qui prennent naissance aux pieds des gorges de Diosso. La deuxième méga séquence datée entre 300 ans B.P. et 100 ans B.P., marque le creusement par les cours d'eau de leurs propres chenaux en cherchant à rétablir un nouveau profil d'équilibre au cours duquel se développent des terrasses. Cette deuxième méga séquence se termine par une reprise normale de la dynamique habituelle des cours d'eau telle que décrite dans la première méga séquence. L'apparition de l'halloysite uniquement dans la première méga séquence (unités 2 et 3) située sous les grandes terrasses (unité 4), suggèrent une hydratation in-situ de la kaolinite héritée de la série des Gorges. Cette évolution de la sédimentation et des paléoenvironnements est en rapport avec la réhumidification du climat à 500 ans B.P. telle que décrite par Helenga et al. (1992b, 2001).

Mots-clés : Sédiments, méga séquences, marécages, fluviatile, Holocène récent, baie de Loango 


\title{
Sedimentology, Grain-size, Sequences Analyses and Palaeoenvironments of Late Holocene Deposit of the Loango Bay in the Kivesso Sector (Republic of Congo)
}

Miyouna Timothée, Docteur Maître-Assistant

Université Marien NGOUABI, Faculté des Sciences et Techniques,

Laboratoire des Géosciences, République du Congo

Elenga Hilaire, Maître de Conférences

Centre de Recherches Géologiques et Minières (CRGM),

Brazzaville, République du Congo

Essouli Olivier Florent, Docteur Maître-Assistant

Université Marien NGOUABI, Faculté des Sciences et Techniques, Laboratoire des Géosciences, Brazzaville, République du Congo

Koubetchi Tsimba Jeannalpha Dorlya, Université Marien NGOUABI, Faculté des Sciences et Techniques, Laboratoire des Géosciences, Brazzaville, République du Congo

Malounguila-Nganga Dieudonné Maurice,

Centre de Recherches Géologiques et Minières (CRGM),

Brazzaville, République du Congo

Makoundi Issouessou Queen Dietrich,

Université Marien NGOUABI, Faculté des Sciences et Techniques, Laboratoire des Géosciences, Brazzaville, République du Congo

Boudzoumou Florent, Maître de Conférences

Institut de Recherche en Sciences Exactes et Naturelles (IRSEN),

Brazzaville, République du Congo

\begin{abstract}
The sedimentological study, grain-size analyses, mineralogical and sequences analyses coupled with ${ }^{14} \mathrm{C}$ radiocarbon has been achieved in the Loango bay, sector of Kivesso. The paper focuses on understanding the nature, the origin, the mechanism of transportation, the palaeoenvironments of sedimentation as well as the sequential organisation of the sedimentary late Holocene deposit of the Loango bay in the Kivesso sector. The study reveals a swampy zone developed between 500 years B.P. and 300 years B.P. on a coastal sandy barrier, built during the last Holocene regression. This swampy zone occupied the sector of Kivesso and was covered by an ombrophile forest. The swampy zone was progressively filed by sediments coming from the
\end{abstract}


"Serie des Cirque" of Diosso and transported by small rivers having a contrasted velocity, sometimes torrential. The deposit is organized into meandering fining upward elementary sequences type fluvial. Two mega sequences corresponding to two phases of filling have been evidenced. The first mega sequence dated between 500 years B.P. and 300 years B.P., corresponds to the units 2 and 3. It is characterized with the development of swampy zone and its filling up by the sediments transported by the rivers coming from the bottom of the "Cirques de Diosso". The second mega sequence dated between 300 years B.P. and 100 years B.P., marks the scouring by the rivers of their own channels that developed the terraces. This second mega sequence ends by a normal resumption of the usual rivers dynamic as described in the first mega sequence. The apparition of the halloysite only in the first mega sequence (units 2 and 3), under the big terraces (unit 4), suggest a hydration in-situ of the kaolinite inherited from "Série des Cirques". This evolution of the sedimentation and palaeoenvironments is in accordance with the rehumidification of the climate at 500 years B.P. as described by Helenga et al. (1992b, 2001).

Keywords: Sediments, mega sequences, swamps, fluvial, Late Holocene, Loango bay

\section{Introduction}

Les formations du littoral congolais ont fait l'objet de plusieurs études géologiques (Giresse, 1978 ; Giresse \& Kouyoumontzakis, 1974 ; Giresse \& Moguedet, 1982 ; Giresse \& Le Ribault, 1981; Giresse et al., 1982 ; Malounguila-Nganga et al., 1983 ; Giresse \& Lanfranchi 1984 ; Elenga \& Vincens, 1990 ; Elenga et al., 1996 ; Bertaux et al., 2000) qui ont contribué à leur connaissance pédologique (Schwartz, 1985, 1990), sédimentologique et paléoenvironnementale (Giresse et Kouyoumontzakis, 1974 ; Giresse et Moguedet, 1982 ; Malounguila-Nganga et al., 1983, 1990 ; Peyrot, 1983), paléobotanique et paléoclimatologique (Deschamps et $a l$., 1988 ; Elenga et al., 1992a ; 1992b ; 2001 ; Elenga \& Vinces, 1990). Ces études ont abouti à la reconstitution des anciennes lignes de rivage, des paléoenvironnements au cours du Pléistocène et de l'Holocène en rapport avec les fluctuations climatiques et eustatiques. Cependant, le remplissage sédimentaire holocène de la baie de Loango n'a pas encore fait l'objet d'une étude sédimentologique exhaustive permettant de mieux comprendre l'origine, la nature et l'organisation séquentielle des corps sédimentaires ainsi que leurs paléoenvironnements sédimentaires dans lesquels ils se sont mis en place. Certains auteurs tels que Giresse et Kouyoumontzakis (1974) ; Schwartz (1990) pensent qu'il s'agit des colluvions provenant de l'érosion de la série des Gorges. L'érosion très active des côtes congolaises au cours des trente 
dernières années a mis à l'affleurement des sédiments holocènes jusqu'alors enfouis, qui permettent de nouvelles observations. Ces observations poussent à de nouvelles interprétations d'ordre sédimentologique, dynamique, séquentielle, minéralogique et paléoenvironnemental du remplissage sédimentaire Holocène de la baie de Loango dans le secteur de Kivesso.

Dans ce sens, le but de cette étude est de mieux comprendre l'origine, la nature des sédiments Holocène récents remplissant la baie de Loango, leur organisation séquentielle, les conditions hydrodynamiques de leur sédimentation ainsi que leurs paléoenvironnements sédimentaires.

\section{Contextes Géographique Et Géologique}

\subsection{Contexte géographique}

La zone d'étude se localise à Kivesso dans le département du Kouilou, au Sud-ouest de Pointe-Noire, capital économique de la République du Congo (Figure 1). Kivesso est une petite localité située sur le littoral ponteningrin, sur la route du Bas Kouilou, après le village Matombi en allant vers le pont sur le fleuve Kouilou. La zone d'étude se situe en bordure de la côte, dans la grande baie de Loango. Les coordonnées géographiques sont les suivantes : longitude $11^{\circ} 49^{\prime} 34,05^{\prime \prime}$; latitude $04^{\circ} 36^{\prime} 44,05^{\prime \prime}$; altitude moyenne $+4 \mathrm{~m}$.

Le climat de la zone d'étude est contrasté et il est du type bascongolais, caractérisé par une alternance d'une saison pluvieuse chaude et d'une saison sèche fraîche (Atlas du Congo, ed. J A, Paris, 2001). La saison pluvieuse chaude s'étend sur 8 mois, d'octobre à mai, avec un fléchissement entre les mois de février et mars. La pluviosité est contrastée (Samba-Kimbata et al., 1990). La moyenne annuelle dans la région est de $1211 \mathrm{~mm}$ avec une variabilité interannuelle marquée : la hauteur maximum a atteint $2162 \mathrm{~mm}$ en 2008 alors que le minimum de $500 \mathrm{~mm}$ a été observé entre 1958 et 1978.

Le couvert végétal est constitué d'une mosaïque de forêts et savanes. Le long du littoral on trouve des mangroves dans les marais (marais de Songolo dans la baie de Pointe-Noire), les lagunes et au niveau des estuaires (Kouilou, Noumbi). Ces forêts à mangroves sont constituées de palétuviers genre Rhizophora mangle et avicénia. La savane est plus herbacée, à dominance Loudetia hypparhenia, soit arbustive dominée par Annona Sénégalensis, dont la taille ne dépasse pas $1,5 \mathrm{~m}$; fourré littoral à Manilkara lacera et prairie à Anadelphia sp. Sur le plateau de Hinda, la végétation à savane est dominée par hypparrhenia ruprechtii (Makany, 1964 ; Schwartz, D. et al., 1990). Ce massif forestier et cette savane herbacée ont été progressivement remplacés par une forêt artificielle à grande majorité d'Eucalyptus et par les arbres fruitiers. Cette forêt occupe beaucoup plus le plateau de Hinda. 


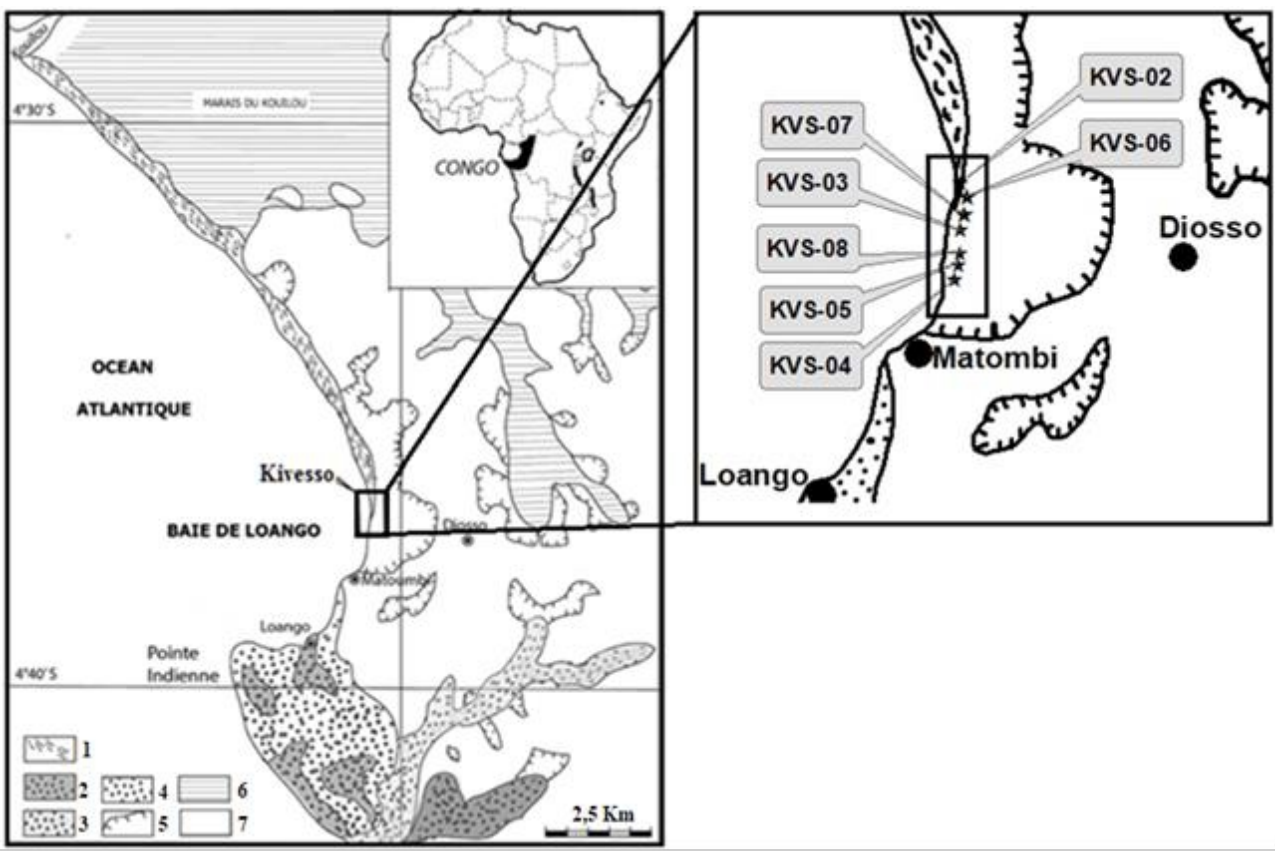

Figure 1. Localisation de la zone d'étude et des points d'observation sur la carte pédologique de la République du Congo (Malounguila-Nganga et al., 2017 modifié)

Légende : (1). Sol sableux hydromorphe de la plaine côtière ; (2). Sol organique développé sur sable ; (3). Sol sableux hydromorphe ; (4). Sol sableux ferralitique ; (5). Sol immature sur colluvion; (6). Zone marécageuse ; (7). Sol ferralitique sur sable argileux de la série des Cirques

Le fleuve Congo est le principal distributeur des sédiments d'origine continentale sur le plateau continental depuis le Cabinda jusqu'au sud du Gabon (Giresse \& Kouyoumontzakis, 1974 ; Giresse et al., 1980). Dans le secteur de Pointe Noire et ses environs les petits cours d'eau côtiers, comme la Songolo dans la baie de Pointe Noire et les rivières Loango et Rouge prennent naissance au pied des Gorgesde Diosso dans la baie de Loango.

Le relief est contrasté, et s'organise autour de trois ensembles morphologiques qui s'élèvent de l'ouest vers l'est. Il s'agit de la plaine côtière, des plateaux côtiers et du massif montagneux du Mayombe. La plaine côtière est légèrement ondulée et séparée de l'océan par des cordons sableux disposés en trois séries parallèles les unes des autres (Giresse, 1981). Les plateaux qui dominent la plaine littorale ont deux aspects. À l'ouest, ils sont tabulaires, légèrement ondulés, par endroits, avec des altitudes comprises entre $130 \mathrm{~m}$ et $145 \mathrm{~m}$, découpés par endroits par des vallées très profondes au fond large et marécageux qui les traversent d'est en ouest (Sitou et al., 1992). À l'est, les plateaux sont plus tourmentés et forment un relief de collines massives aux flancs très inclinés, dont la pente dépasse presque partout $20 \%$. Celles-ci 
culminent à $175 \mathrm{~m}$ et sont dans un désordre apparent (Malounguila-Nganga et al., 1983).

L'évolution paléoclimatique en Afrique centrale met en évidence au Quaternaire quatre grandes phases climatiques qui peuvent être corrélées avec les évolutions de la végétation et des mouvements de transgression et de régression le long du littoral congolais (De Ploey, 1963). De la fin du Pléistocène à l'actuel on $\mathrm{a}$ : une première phase à tendance sèche, le Maluekien, qui s'étend de 70000 ans B.P. à 40000 ans B.P. ; une phase de réhumidification centrée vers 35000 ans B.P., le Njilien ; une phase très aride, le Léopoldvillien, dont le maximum est daté entre 20000 ans B.P. et 18000 ans B.P. Ensuite, une phase de réhumidification du climat, le Kibanguien, qui débute vers 12000 ans B.P. et que l'on divise en Kibanguien A (maximum de la réhumidification, de 12000 ans B.P. à 3-4000 B.P.), et le Kibanguien B, léger assèchement (Elenga et $a l ., 1992$; Bertaux et $a l$. , 2000) qui conduit à une réhumidification du climat dès 500 ans B.P. jusqu'au climat actuel (Malounguila-Nganga et al., 2017).

\subsection{Contexte géologique}

Le socle précambrien est constitué de roches métamorphiques, magmatiques éruptives et volcaniques sur lequel reposent en discordance angulaire $3500 \mathrm{~m}$ à $4000 \mathrm{~m}$ de sédiments d'âge Crétacé à l'Holocène (Grosdidier et $a l .$, 1996). Sur le continent les plateaux de Hinda qui bordent la plaine côtière sont constitués des formations d'âges Plio-pléistocène de la série des Cirques, formées essentiellement des sables à intercalations de niveaux argileux (Desthieux et $a I .$, 1993). Ces sables comprennent de multiples horizons durcis évoluant en sorte de paléosols. La série des Cirques d'origine fluvio-lacustre (Peyrot, 1983) ou fluvio-deltaïque (Callec et al., 2015) est recouverte par du sable fin, argileux et silteux de couleur jaune ocre de la Formation de couverture d'origine éolienne (Miyouna et al., 2016). Les formations du Crétacé affleurent en bordure du Mayombe où elles constituent les formations de contact et au niveau des pointes le long de la côte.

\section{Méthode d'étude}

La méthode de l'étude a consisté en une étude sur terrain, au laboratoire et à l'analyse et interprétation des résultats. L'étude sur le terrain a consisté à décrire les affleurements rencontrés sur la baie sur une distance de 2,200 km, à lever des logs et à échantillonner. L'échantillonnage s'est fait du bas vers le haut afin d'éviter toute contamination. Sept (7) logs ont été levés (Figure 1) : Kivesso 2, Kivesso 3, Kivesso 4, Kivesso 5, Kivesso 6, Kivesso 7, Kivesso 8. L'étude de laboratoire a porté sur les échantillons des affleurements de Kivesso 4 (KVS4) et Kivesso 8 (KVS8) qui représentent la 
succession verticale la plus complète dans le secteur. Les analyses suivantes ont été faites :

- le fractionnement granulométrique à la pipette de Robinson pour déterminer la proportion relative de sable grossier, sable fin, silts et d'argile dans chaque échantillon. Il a été effectué au laboratoire de l'Institut National de Recherche en Sciences Exactes et Naturelles (IRSEN) de Pointe Noire ;

l'analyse granulométrique par tamisage à sec de la fraction sableuse, la minéralogie et la morphoscopie ont été faites au laboratoire des Géosciences de la Faculté des Sciences et Techniques de l'Université Marien NGOUABI de Brazzaville. Le tamisage a été fait à l'aide d'une colonne de six tamis à mailles décroissantes vers le bas en progression AFNOR. Le refus de chaque tamis a été pesé et cumulé ; il a permis de tracer les courbes granulométriques cumulatives à partir desquelles nous avons déterminé les indices granulométriques en vue de calculer les paramètres granulométriques tels que la moyenne, l'écart-type et l'asymétrie selon les formules de Folk et Ward (1957). Les valeurs de ces paramètres ont été réparties le long de chaque profil afin de suivre leur évolution verticale ;

l'étude morphoscopique a été faite au laboratoire des Géosciences de la Faculté des Sciences et Techniques de l'Université Marien NGOUABI selon la méthode développée par Tricart (1965) sur les fractions 0,500 mm et $0,250 \mathrm{~mm}$ à l'aide d'une loupe binoculaire. Son principe consiste à examiner la forme, le type et l'état de la surface des grains de quartz, dans le but d'apporter des renseignements sur l'environnement de sédimentation et le mode de transport;

- $\quad$ l'analyse chimique a consisté au dosage d'azote, carbone total et de la matière organique dans chaque échantillon. Elle a été faite au laboratoire de 1'Institut National de Recherche en Sciences Exactes et Naturelles (IRSEN) de Pointe-Noire. Le dosage de l'azote total a été fait selon la méthode de Kjeldal (1883) et celui du carbone total et de la matière organique par la méthode de Walkley et Black (1934). Les niveaux riches en matière organique ont été préférentiellement sélectionnés pour les datations au radiocarbone $\left({ }^{14} \mathrm{C}\right)$ au laboratoire Posnan de Pologne. La calibration a été faite en utilisant la courbe de calibration de l'hémisphère nord à l'aide du logiciel Calib 5.0.02 (Stuiver \& Reimer, 1993).

\section{Résultats \\ 4.1. Résultats de terrain \\ 4.1.1. Description du profil de Kivesso 4}

Le log de Kivesso 4 (KVS4) a été réalisé au point de coordonnées géographiques suivantes : longitude $11^{\circ} 49^{\prime} 35^{\prime \prime}$, latitude $04^{\circ} 36^{\prime} 53,3^{\prime \prime}$ et altitude de 5,9 m. Il montre du bas vers le haut cinq unités lithologiques. 
L'unité $\mathbf{1}(\mathbf{0} \mathbf{~ m}$ à $\mathbf{0 , 8 0} \mathbf{~ m})$ n'affleure que sur $0,80 \mathrm{~cm}$. Elle se trouve en grande partie sous le niveau marin actuel. Elle est constituée de sable grossier blanc, homogène, bien classé devenant beige vers le bas ;

L'unité $2(\mathbf{0 , 8 0} \mathbf{m}$ à $\mathbf{1 , 6 7} \mathbf{m})$ est constituée de bas en haut de :

- $\quad 0,37 \mathrm{~m}$ d'argile plastique marron contenant un lit de $13 \mathrm{~cm}$ de tourbe noire, à racines et écorces, stratifiées parallèlement au litage ;

- $0,30 \mathrm{~m}$ de sable blanc moyen bien classé à litages obliques en feston et montrant une alternance des lits clairs de sable blanc avec des lamines noires contenant des minéraux lourds à prédominance ilménite ;

- $\quad 0,20 \mathrm{~m}$ d'argile plastique ocre.

L'unité 3 (1,67 $\mathbf{m}$ à 3,29 $\mathbf{m})$ est constituée du bas vers le haut de :

- $\quad 0,16 \mathrm{~m}$ de sable beige, moyen mal classé ;

- $\quad$ 0,10 m d'argile silteuse grise renfermant des micro rides blanchâtres ;

- $\quad 0,17 \mathrm{~m}$ de sable fin à moyen gris blanchâtre et stratifié, composé d'une alternance de lits de sable blanc fin à moyen avec des lamines de sable silteux sombre ;

- $\quad 0,50 \mathrm{~m}$ de sable gris moyen ;

- $\quad 0,50 \mathrm{~m}$ de sable gris blanc, grossier à moyen, stratifié montrant une alternance de lits de sable gris de 0,2 m d'épaisseur avec des lits de sable grossier de $3 \mathrm{~cm}$ d'épaisseur ;

- $\quad 0,10 \mathrm{~m}$ d'argile silteuse marron ;

- $\quad 0,20 \mathrm{~m}$ de sable fin argileux gris blanchâtre ;

- $\quad 0,10 \mathrm{~m}$ d'argile marron ;

- $\quad 0,25 \mathrm{~m}$ de sable fin à moyen, jaune et homogène ;

- $0,70 \mathrm{~m}$ d'argile ocre plastique durcie et qui contient des fentes de retrait ;

- $\quad 0,19 \mathrm{~m}$ de sable fin ocre en banc massif ;

- 0,40 m d'argile ocre renfermant des racines et des écorces longeant la surface de stratification ;

- 0,34 $\mathrm{m}$ de sable fin argileux rouge ocre bien classé et homogène devenant légèrement grossier dans la partie supérieure et à altération blanchâtre. Latéralement, il présente des lentilles de sable fin et blanc qui semblent fossiliser les rides dissymétriques de courant. En allant vers le nord, à 200 mètres de ce log, ce niveau devient de plus en plus grossier et graveleux ;

- $\quad 0,20 \mathrm{~m}$ d'argile rouge ocre plastique. 
L'unité $\mathbf{4}(\mathbf{3 , 2 9} \mathrm{m}$ à $\mathbf{4 , 8} \mathrm{m})$ est constituée de bas en haut de :

- $\quad 0,55 \mathrm{~m}$ de sable moyen rouge ocre contenant dans la partie supérieure des lentilles de sable blanc grossier en forme de gouttières d'érosion ;

- $\quad$ 0,80 m de sable moyen jaune blanchâtre reposant sur une lamine de sable silteux rouge. Sa partie supérieure présente des rides de courant fossilisées par des fines lamines d'argile silteuse rouge ;

- 0,23 m de sable moyen jaune blanchâtre surmontant une lamine de sable argileux rouge ocre ;

- $\quad 0,26 \mathrm{~m}$ de sable blanc moyen très friable et très pauvre en argile ;

- $\quad 0,22 \mathrm{~m}$ de sable argileux gris ;

- $\quad 0,10 \mathrm{~m}$ de sable moyen blanc friable ;

- $\quad$ 0,40 $\mathrm{m}$ de sable argileux gris riche en racine de graminée.

L'unité $5(\mathbf{4 , 8} \mathrm{m}$ à $\mathbf{5 , 5 8} \mathrm{m})$ est constituée du bas vers le haut :

- $\quad 0,24 \mathrm{~m}$ de sable blanc fin à moyen homogène ;

- $\quad 0,20 \mathrm{~m}$ de sol humifère actuel. 


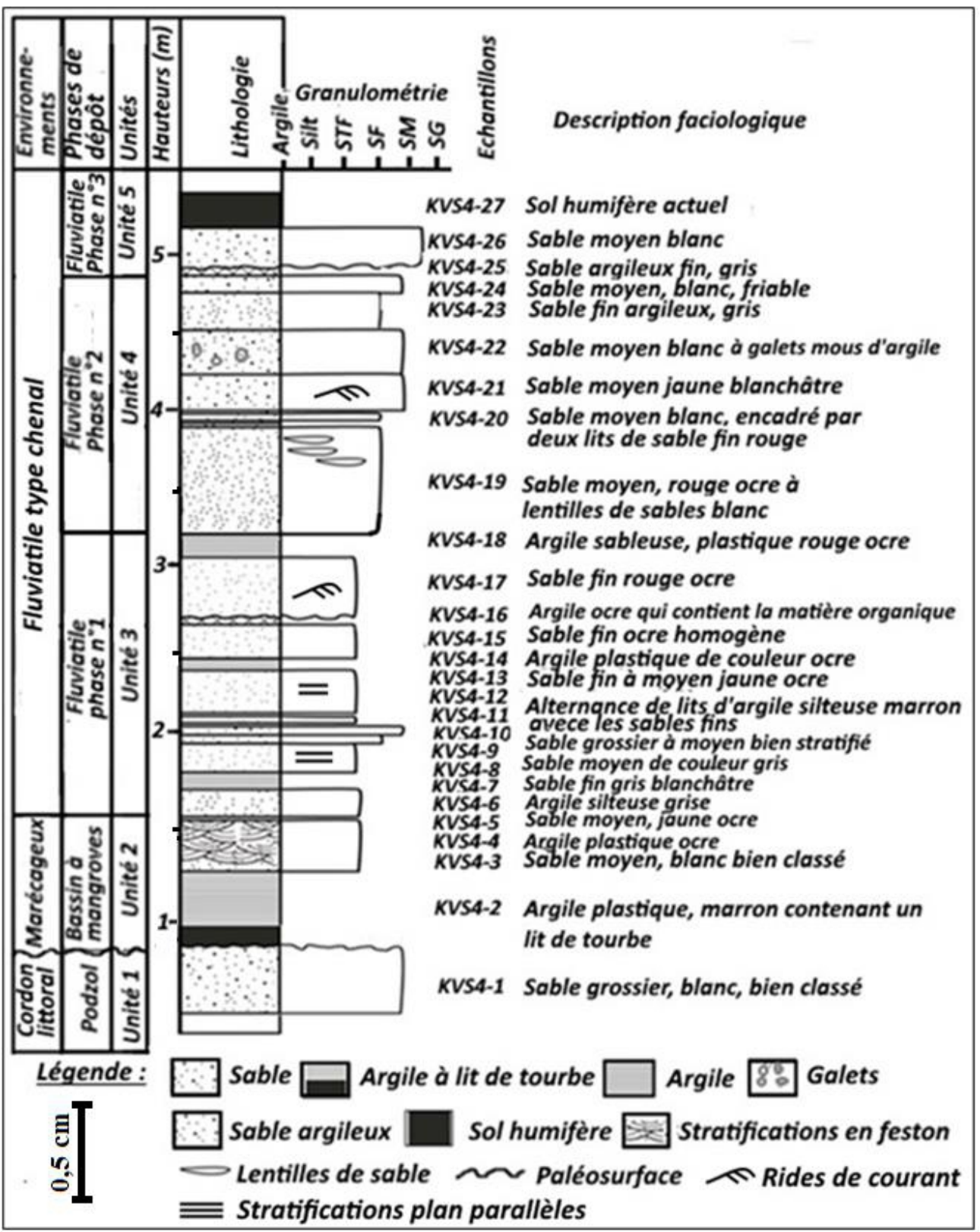

Figure 2. Log lithologique de Kivesso 4

\subsubsection{Description du profil de Kivesso 8}

Le profil de Kivesso 8 (KVS8) a été réalisé au point des coordonnées géographiques : latitude : $04^{\circ} 36^{\prime} 44,05^{\prime \prime}$; longitude : $11^{\circ} 49^{\prime} 34,05^{\prime \prime}$; altitude : 9,69 m. Il montre du bas vers le haut deux grandes unités qui correspondent aux unités 4 et 5 décrites à KVS4. 
L'unité $4(0,38 \mathrm{~m}-5 \mathrm{~m})$ est constituée de bas en haut de :

- $\quad$ 0,62 $\mathrm{m}$ de sables fins rouge ocre contenant des lamines d'argile beige à rouge ocre. Ce sable renferme de petite concrétion latéritique et de petits galets mous rouge ocre ;

- 0,18 m de sables rouges blanchâtres moyen à grossier, légèrement graveleux, à stratifications plan parallèles diffus ;

- 0,50 m de terrasse alluviale constituée majoritairement de blocs, graviers et granules de latérite très dure associés aux blocs et galets arrondis d'argilite rouge, aux graviers de quartzite blanc. La matrice est sableuse légèrement argileuse (Figure 4, photo e) ;

- $\quad 0,15 \mathrm{~m}$ de sables rouges blanchâtres, moyens à grossiers d'apparence homogène à traces de racines actuelles ;

- $\quad 0,25 \mathrm{~m}$ de sables rouges ocre, fins, légèrement argileux, homogène à petites lentilles de sable beige dans la partie sommitale qui fossilisent les rides de courant. Ce sable est raviné au sommet ;

- $\quad$ 0,14 m de terrasse alluviale composée de blocs et granules de latérite bréchique, de galets et graviers blancs de quartzite, le tout emballé dans une matrice sablo-argileuse rouge ocre. L'épaisseur de cette terrasse varie latéralement jusqu'à atteindre $1,30 \mathrm{~m}$. La taille des galets de quartzite atteint $10 \mathrm{~cm}$, par contre les blocs de latérite atteignent jusqu'à $30 \mathrm{~cm}$ d'épaisseur (Figure 4, photo e) ;

- $\quad 0,36 \mathrm{~m}$ de sable graveleux de couleur rouge ocre légèrement blanchâtre à fins niveau centimétrique de gravier. Ce sable graveleux est raviné au sommet ;

- $\quad$ 0,13 m de sable beige moyen à rides de courant dans la partie basale et à stratifications plan parallèles au sommet. Les rides et les stratifications sont marquées par les minéraux lourds. Ce sable montre de nombreuses gouttières d'érosion remplies de sable beige à stratifications progradantes vers le sud tantôt vers le nord (Figure 4, photo c) ;

- 0,17 m de sables moyens beige. Ce sable débute par un fin niveau graveleux de $2 \mathrm{~cm}$ et il est constitué de granules de quartzite et de latérite ;

- $\quad 0,18 \mathrm{~m}$ de sables moyens à grossiers de couleur rouge ocre débutant à sa base par $1 \mathrm{~cm}$ de granules de latérite et de quartzite ;

- 0,13 m de sables blancs rougeâtres moyens à stratifications plan parallèles horizontales. Ce sable sus-jacent ravine le sable rouge ocre. On y observe également de petites rides de courant centimétriques. La stratification est bien marquée par les lits de minéraux lourds de couleurs sombres (Figure 4, photo b) ;

- $0,13 \mathrm{~m}$ de sable fin, rouge ocre à beige, d'apparence homogène, à stratifications plan parallèles subhorizontales ; 
- $0,20 \mathrm{~m}$ d'argile très plastique grisâtre à la base et rougeâtre au sommet ;

- 0,70 m de sables moyens blancs jaunâtres à stratifications plan parallèles diffus, parfois matérialisées par les minéraux lourds ;

- $\quad 0,30 \mathrm{~m}$ d'argile rouge très plastique à débris de matière organique à la base ;

- $\quad 0,18 \mathrm{~m}$ de sable blanc rougeâtre, fin. Il renferme de lamines d'argile rouge ;

- $\quad 0,20 \mathrm{~m}$ d'argile très plastique, de couleur rouge ;

- $\quad 0,10 \mathrm{~m}$ de sable rouge ocre à beige, fin et légèrement argileux ;

- $\quad 0,47 \mathrm{~m}$ d'argile rouge ocre, très plastique disposée en plaquette de 4 à $10 \mathrm{~cm}$ d'épaisseur. À l'interface des plaquettes se trouvent des débris végétaux représentés par des petites tiges, des petites feuilles et des herbes encore bien conservés ;

- 0,14 m d'argile plastique constituée par une alternance de lamines rouges ocres et de lamines grisâtres plus ou moins silteuses.

L'unité 5 (5 m-9,6 m) est constituée de bas en haut de :

- 0,12 m de sable blanc moyen à rides de courant soulignées par des minéraux lourds ;

- $\quad 0,60 \mathrm{~m}$ d'argile beige très plastique ;

- $\quad$ 0,80 m de sable blanc jaunâtre, moyen à rides de courant dans la partie sommitale et à stratifications obliques progradantes vers le nord ;

- $\quad 0,80 \mathrm{~m}$ d'argile plastique rouge ocre à la base renfermant des débris de matière organique ;

- $\quad 0,24 \mathrm{~m}$ de sable rouge blanchâtre à beige, moyen à fin contenant des lentilles d'argile rouge ;

- 0,13 m d'argile plastique rouge ocre à beige à débris de racines actuelles ;

- $\quad 0,29 \mathrm{~m}$ de sable blanc fin à petits galets d'argile rouge remaniée ;

- $\quad 0,30 \mathrm{~m}$ d'argile plastique rouge ocre ;

- $\quad 0,11 \mathrm{~m}$ de sable blanc fin d'apparence homogène ;

- $\quad 0,60 \mathrm{~m}$ d'argile plastique rouge ocre ;

- 0,19 m de sable de couleur beige moyen, homogène légèrement argileux. Ce sable est légèrement rubéfié par la pédogenèse actuelle ;

- $\quad 0,40 \mathrm{~m}$ d'argile compacte et plastique de couleur rouge ocre ;

- 0,68 m de sable argileux fin, de couleur beige à gris verdâtre d'apparence homogène ;

- $\quad 0,15 \mathrm{~m}$ de sable blanc grisâtre, moyen légèrement argileux ;

- $\quad 0,40 \mathrm{~m}$ de sable moyen grisâtre et argileux ; 
- 0,22 $\mathrm{m}$ de sable moyen rouge ocre à galets de couleur blanchâtre d'argile sableuse ;

- $\quad 0,30 \mathrm{~m}$ de sable moyen beige à traces d'oxyde de fer résultants de la pédogenèse actuelle ;

- $\quad$ 0,17 m de sable rouge ocre, à rides de courant. Ce sable contient des petites gouttières d'érosion remplies par du sable moyen de couleur blanche ;

- 0,33 $\mathrm{m}$ de sable blanc ocre, moyens d'apparence homogène, localement bioturbé. On y note quelques concrétions ferrifères d'origine pédogénétique actuelle ;

- $\quad 0,57 \mathrm{~m}$ de sables fins débutant par $9 \mathrm{~cm}$ de sables grossiers à graveleux à la base. Ce sable de couleur rouge ocre, d'apparence homogène renferme des racines actuelles ;

- $0,30 \mathrm{~m}$ de sol très fin, noire grisâtre, sablo-argileux et humifère, renfermant des terriers. 


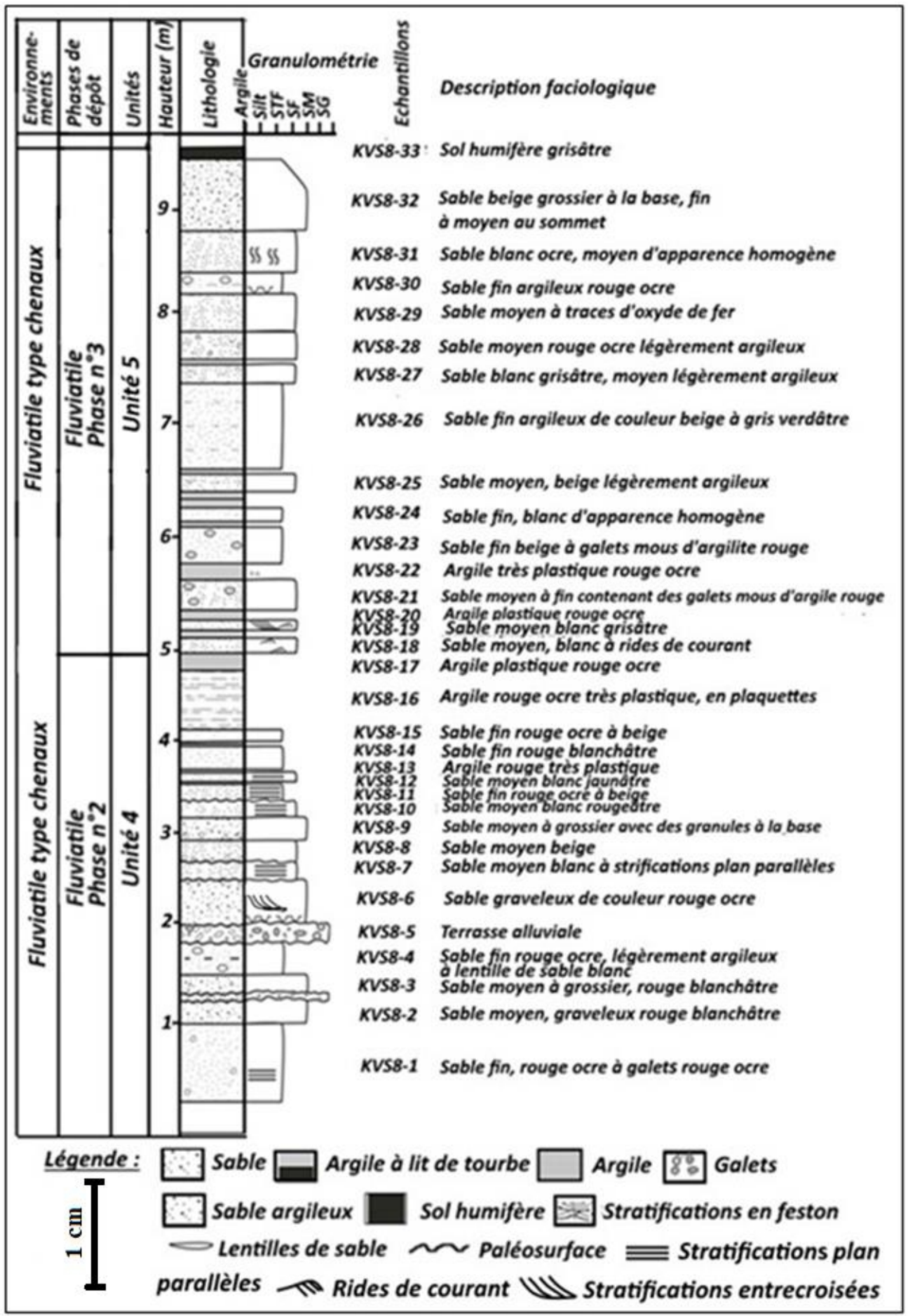

Figure 3. Log lithologique de Kivesso 8 


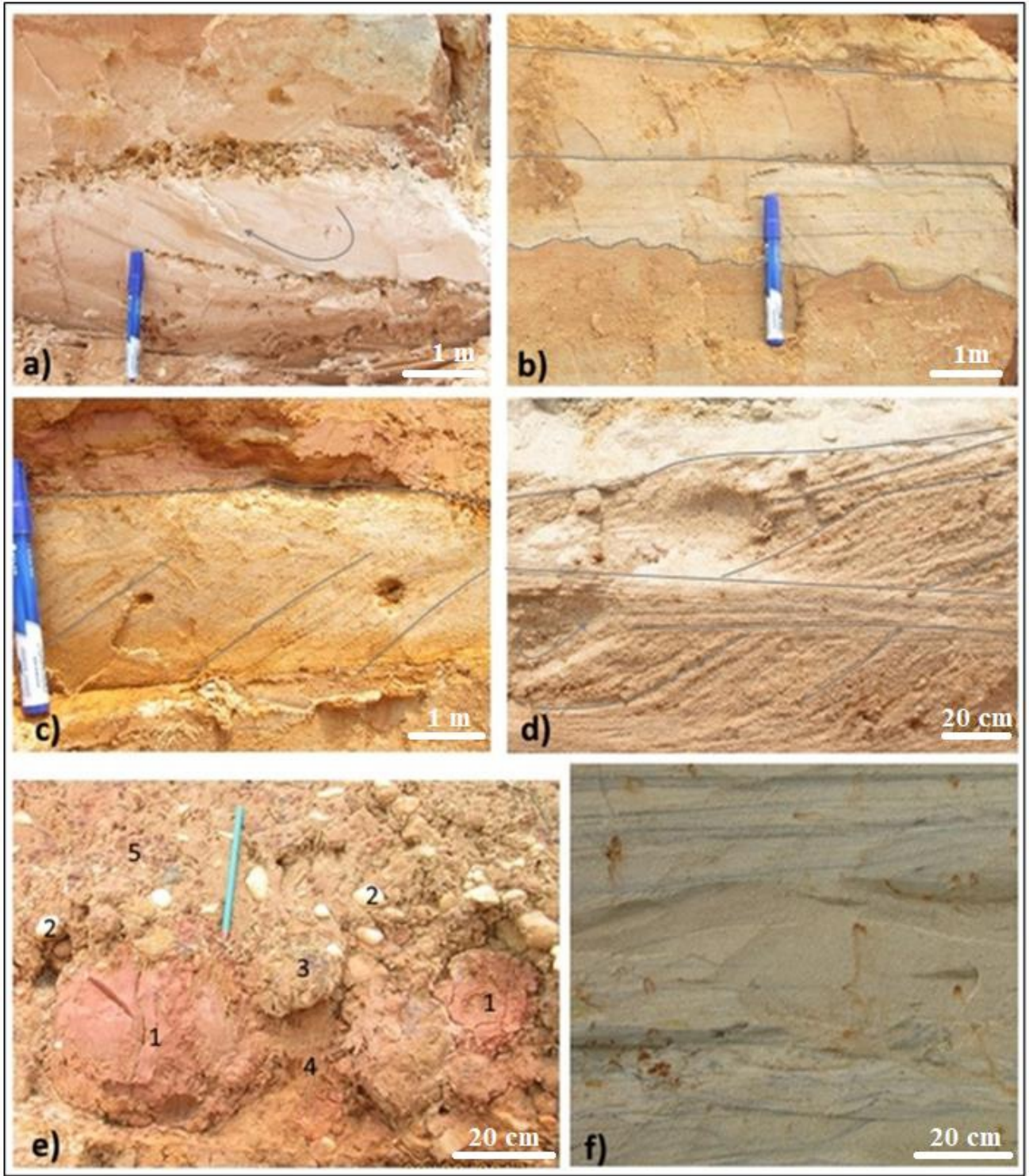

Figure 4. Lithofaciès observés à Kivesso 8.

Légende : a) Sable graveleux rouge lie-de-vin à stratification obliques arquées marquées par des graviers qui sont grossiers à la base et devenant fin progressivement vers le haut. La flèche courbe indique le sens du courant hélicoïdale; $\boldsymbol{b})$ gouttière d'érosion remplie par du sable beige à stratifications plan parallèles; c) litages obliques parallèles, progradantes, structurant un sable beige moyen; d) Stratifications entrecroisées dans un sable grossier et graveleux rouge ocre ; $\boldsymbol{e})$ Terrasse alluviale constituée de galets d'argilite rouge (1), de galets blanc de quartzite (2), de blocs pluri centimétriques de latérite (3), de granules de latérite (5) le tout emballé dans une matrice sablo-argileux rouge ocre (4);f) Sable gris à stratifications entrecroisées en feston à la base et stratification plan parallèle au sommet. 


\subsection{Résultats des analyses sédimentologiques}

\subsubsection{Fractionnement à la pipette de Robinson}

Le fractionnement à la pipette de Robinson montre que les sédiments holocènes affleurant dans le secteur de Kivesso sont essentiellement des sables et des argiles (Tableau 1). La proportion en argile varie entre $1,45 \%$ et $72 \%$ ; celle en silts varie entre $0,165 \%$ et $20,24 \%$; entre $11 \%$ et $76 \%$ celle en sable fin et entre $0,90 \%$ et $85 \%$ celle en sable grossier.

Tableau 1. Proportion en argile, silts, sable fin et sable grossier dans les sédiments $d u$ remplissage holocène de la baie de Loango, profile de Kivesso 4 (KVS4)

\begin{tabular}{|c|c|c|c|c|c|}
\hline Echantillons & Hauteur (m) & Argile (\%) & Silt (\%) & Sable fin (\%) & Sable grossier (\%) \\
\hline KVS 4 - 1 & $+0,70$ & 0,00 & 0,17 & 13,36 & 85,80 \\
\hline KVS $4-2$ & $+1,20$ & 72,30 & 6,82 & 11,68 & 9,20 \\
\hline KVS 4- 3 & $+1,50$ & 3,90 & 7,41 & 76,31 & 11,45 \\
\hline KVS 4- 4 & $+1,63$ & 9,40 & 4,70 & 46,71 & 38,00 \\
\hline KVS $4-5$ & $+1,70$ & 7,40 & 4,70 & 48,71 & 38,00 \\
\hline KVS $4-6$ & $+1,80$ & 48,12 & 20,30 & 21,13 & 10,45 \\
\hline KVS $4-7$ & $+1,88$ & 3,90 & 2,52 & 40,15 & 52,72 \\
\hline KVS $4-8$ & $+1,95$ & 11,65 & 6,09 & 48,28 & 33,33 \\
\hline KVS $4-9$ & $+2,03$ & 6,35 & 4,26 & 44,97 & 43,69 \\
\hline KVS $4-10$ & $+2,07$ & 52,12 & 18,30 & 22,13 & 7,45 \\
\hline KVS $4-11$ & $+2,10$ & 4,05 & 3,39 & 62,65 & 29,10 \\
\hline KVS $4-12$ & $+2,13$ & 56,12 & 12,30 & 23,13 & 8,45 \\
\hline KVS $4-13$ & $+2,30$ & 8,75 & 3,76 & 38,89 & 47,89 \\
\hline KVS $4-14$ & $+2,45$ & 44,75 & 19,18 & 33,26 & 0,90 \\
\hline KVS $4-15$ & $+2,57$ & 10,05 & 5,00 & 44,48 & 39,41 \\
\hline KVS 4 - 16 & $+2,68$ & 43,65 & 18,18 & 34,26 & 3,91 \\
\hline KVS $4-17$ a & $+2,90$ & 9,00 & 5,21 & 38,61 & 46,41 \\
\hline KVS 4 - 18 & $+3,15$ & 41,00 & 20,24 & 27,27 & 8,79 \\
\hline KVS 4 - 19 a & $+3,60$ & 7,35 & 4,18 & 32,78 & 55,30 \\
\hline KVS 4 - 20 & $+3,92$ & 3,05 & 2,43 & 46,75 & 47,14 \\
\hline KVS $4-21$ & $+4,12$ & 2,45 & 1,46 & 27,66 & 67,82 \\
\hline KVS $4-22$ & $+4,40$ & 1,45 & 0,99 & 24,33 & 72,81 \\
\hline KVS $4-23$ & $+4,60$ & 9,55 & 5,73 & 48,85 & 35,01 \\
\hline KVS $4-24$ & $+4,78$ & 3,70 & 2,49 & 36,77 & 56,50 \\
\hline KVS $4-25$ & $+4,85$ & 20,15 & 10,90 & 45,64 & 22,50 \\
\hline KVS $4-26$ & $+5,00$ & 3,20 & 0,99 & 20,59 & 74,50 \\
\hline
\end{tabular}


La répartition de ces proportions dans le diagramme des sédiments fins de Krumben (1962) (Figure 5) montre quatre faciès suivants par ordre d'importance décroissant : faciès sableux, faciès argileux, faciès, faciès argilosilto-sableux et le faciès sablo-argileux.

L'évolution verticale de ces proportions le long du profil de KVS 4 (Figure 6) montre que la proportion en argile est très faible dans le cordon sableux situé à la base du profil. Ensuite, elle augmente brutalement à 72,3\% à $1,2 \mathrm{~m}$ puis baisse à $3,90 \%$ à $1,5 \mathrm{~m}$ avant d'augmenter à nouveau à $48,12 \%$ à $1,8 \mathrm{~m}$. Elle baisse à nouveau à $3,90 \%$ à $1,88 \mathrm{~m}$, reste faible jusqu'à $2,07 \mathrm{~m}$ et évolue ensuite en dents de scie entre $56,12 \%$ et 4,05\% de 2,7 m à $3,15 \mathrm{~m}$. À partir de 3,6 m, la proportion en argile chute d'abord brutalement à 7,35\%, puis progressivement jusqu'à atteindre $1,45 \%$ à $4,4 \mathrm{~m}$ avant de remonter à $20,15 \%$ à 4,85 m et baisser enfin à $3,20 \%$ au sommet du profil.

La proportion en silts, très faible dans le cordon sableux situé à la base du profil, augmente progressivement à $7,41 \%$ à $1,5 \mathrm{~m}$ puis baisse à $4,7 \%$ entre $1,63 \mathrm{~m}$ et $1,8 \mathrm{~m}$. Cette proportion augmente à $20,30 \%$ à $1,8 \mathrm{~m}$, puis elle chute à $2,52 \%$ à $1,88 \mathrm{~m}$; elle reste faible jusqu'à $2,03 \mathrm{~m}$ avant d'augmenter considérablement et évoluer en dents de scie entre $18,30 \%$ et $4,26 \%$ de 2,07 $\mathrm{m}$ à $3,15 \mathrm{~m}$. Ensuite, elle chute d'abord à $4,18 \%$ à $3,6 \mathrm{~m}$ puis baisse progressivement jusqu'à atteindre $0,99 \%$ à $4,4 \mathrm{~m}$. Enfin, elle augmente à 10,9 $\%$ à 4,85 m avant de baisser à $0,99 \%$ au sommet du profil.

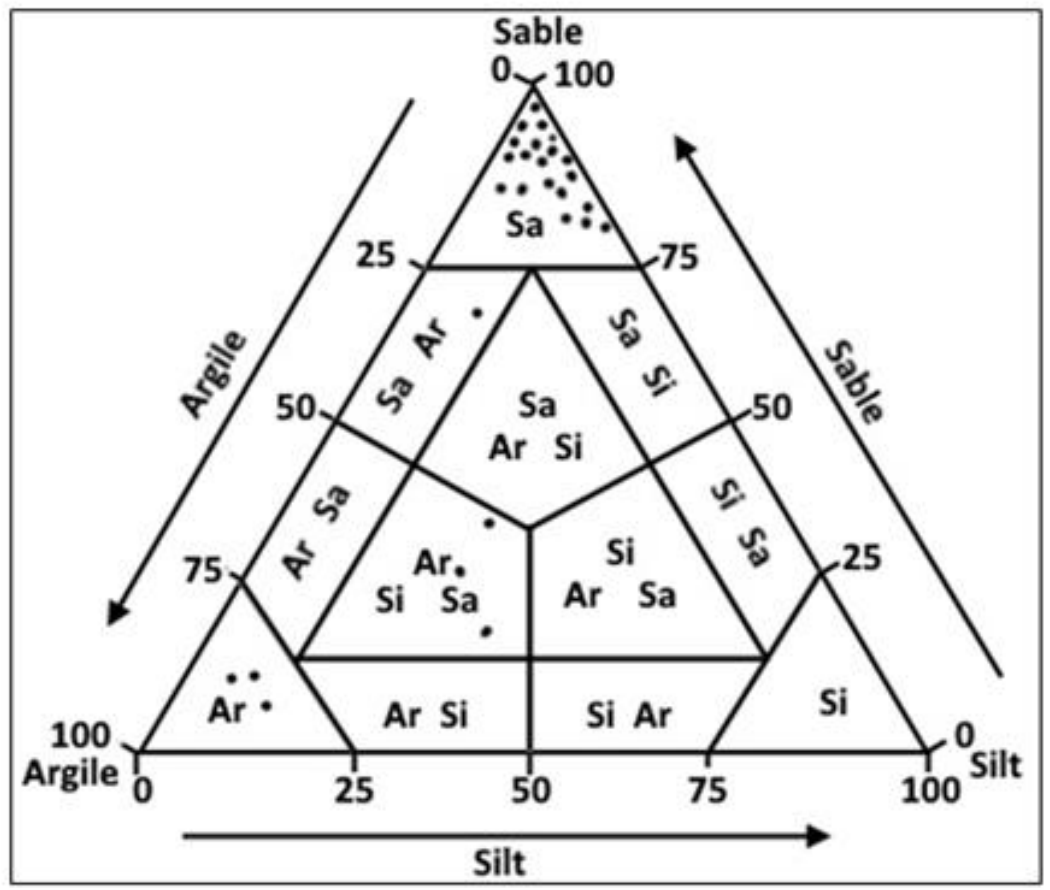

Figure 5. Diagramme ternaire de la dénomination granulométrique des sédiments fins $d u$ profil de Kivesso 4 
La proportion en sable fin, faible de $0,7 \mathrm{~m}$ à $1,2 \mathrm{~m}(11,68 \%)$ augmente considérablement à $76,31 \%$ à $1,5 \mathrm{~m}$, puis décroît progressivement jusqu'à $21,13 \%$ à $1,8 \mathrm{~m}$. Elle augmente à nouveau jusqu'à atteindre $48,28 \%$ à 1,95 $\mathrm{m}$ puis baisse à $22,13 \%$ à $2,07 \mathrm{~m}$. À partir de $2,07 \mathrm{~m}$, elle augmente considérablement à $62,65 \%$ à $2,1 \mathrm{~m}$ puis chute et évolue en dents de scie entre $23,13 \%$ et $27,27 \%$ de $2,13 \mathrm{~m}$ à $3,15 \mathrm{~m}$. Elle augmente progressivement à $46,75 \%$ à $3,92 \mathrm{~m}$ avant de baisser à $24,33 \%$ à $4,4 \mathrm{~m}$. Enfin, elle augmente à $48,85 \%$ à $4,6 \mathrm{~m}$ avant de décroître à $20,59 \%$ au sommet du profil à $5 \mathrm{~m}$.

Par contre, la proportion en sable grossier, élevée $(85,80 \%)$ dans le cordon sableux de base, chute considérablement à $9,2 \%$ à $1,2 \mathrm{~m}$, puis augmente progressivement à $38 \%$ à $1,7 \mathrm{~m}$ avant de décroître à nouveau à $10,45 \%$ à $1,8 \mathrm{~m}$. Elle augmente à nouveau à $52,72 \%$ à $1,88 \mathrm{~m}$, puis baisse progressivement à jusqu'à $7,45 \%$ à $2,07 \mathrm{~m}$. De là, elle augmente encore à $29,10 \%$ à $2,1 \mathrm{~m}$ avant de baisser encore et d'évoluer en dents de scie entre 0,9 $\%$ et $46,10 \%$ de $2,13 \mathrm{~m}$ à $3,15 \mathrm{~m}$. A partir de 3,15 m, la proportion en sable grossier augmente progressivement jusqu'à atteindre $72,81 \%$ à $4,4 \mathrm{~m}$ puis chute à $22,5 \%$ à $4,85 \mathrm{~m}$ avant d'augmenter à $74,5 \%$ au sommet du profil à 5 m.

Cette évolution verticale des proportions en argiles, silts, sable fin et sable grossier confirme les différentes unités lithologiques mises en évidence lors de l'étude sur le terrain.

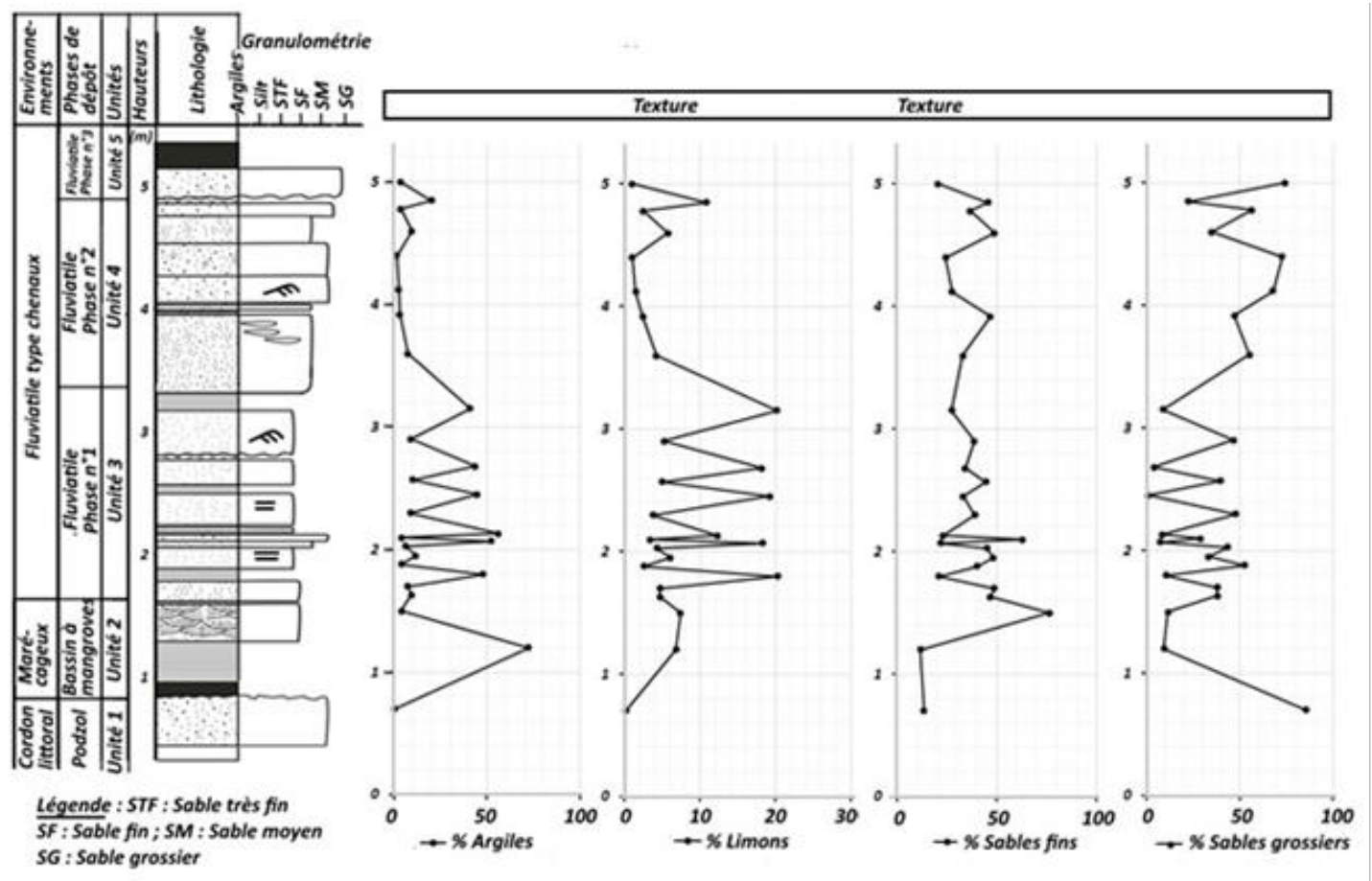

Figure 6. Évolution verticale des proportions en argile, silt, sable fin et en sable grossier le long du profil de KVS 4 


\subsubsection{Granulométrique des sables}

Les courbes granulométriques cumulatives de KVS 8 (Figure $7 c$ et d) et celles de KVS 4 (Figure $7 a$ et $b$ ) sont essentiellement sigmoïdales, régulières, excepté les courbes des échantillons KIVS8-5, KVS8-14 qui sont irrégulières à deux paliers. La pente des courbes est généralement régulière, moyennement redressée et étalée entre 1 et 2,8 unités phi, excepté les courbes des échantillons KVS4-3, KVS4-14 et KVS4-18 qui sont hyperboliques tronquées du côté des fins, elles ont une pente régulière moyennement redressée. L'étalement de la pente de ces différentes courbes indique des sables à granulométrie variable, moyennement à assez bien classés, contenant essentiellement un seul stock granulométrique dominant. Par contre les courbes à deux paliers indiquent des sables mal classés, contenant deux stocks granulométriques. Ce sont des sables mal classés.

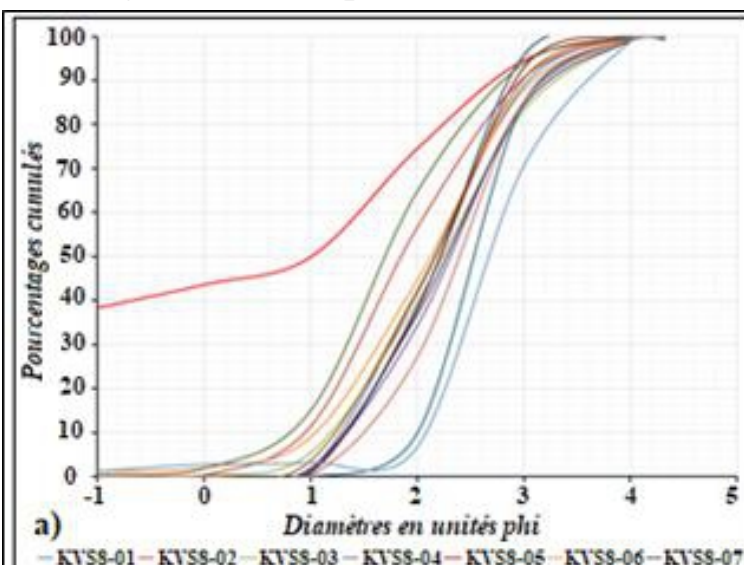

- KTSS-01-KTSS-02 - KTSS-03-KTS8-04-KTSS-05 - KTSS-06-KTSS-07 -KTSS-0S - KTSS-09-KTSS-10 - KTSS-11 - KTSS-12 - KTSS-14-KTSS-15

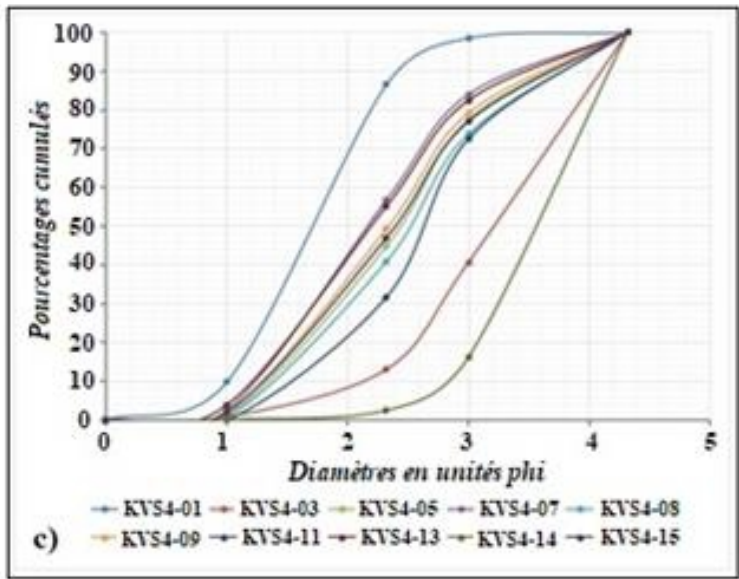

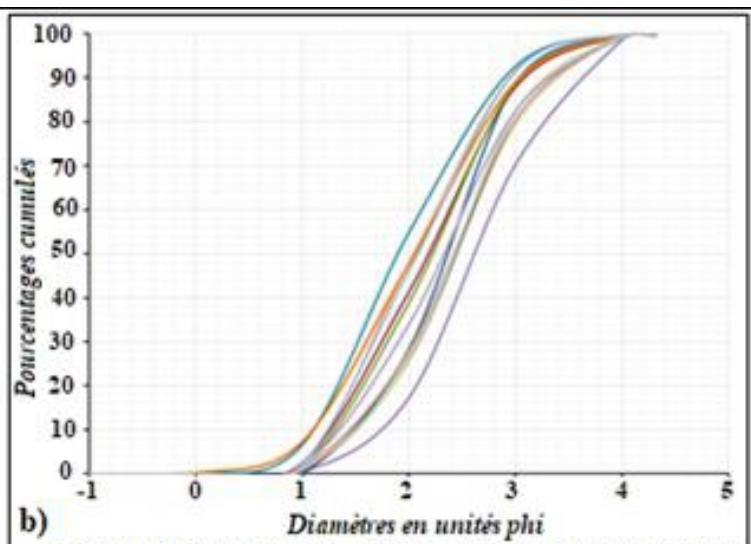

-KTSS-18 - KTSS-19 - KTSS-21-KTSS-23-KTSS-24-KTSs-25-KTSS-26 - KTSS-27 - KTSS-28 - KTSS-29-KTSS-30 - KTSS-31-KTS8-32

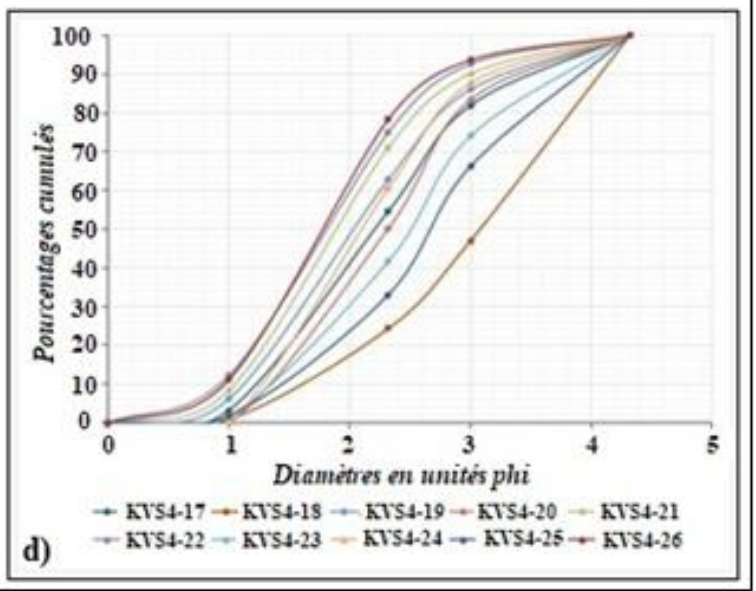

Figure 7. Courbes cumulatives de Kivesso $8(a, b)$ et Kivesso $4(c, d)$

La Figure 8 illustrant la variation verticale des différents paramètres granulométriques le long du log lithologique de KVS 8 montre que : 
- $\quad$ la valeur du percentile Q5 augmente d'abord de 0,440 $\mathrm{mm}$ à 0,620 $\mathrm{mm}$ de $0,7 \mathrm{~m}$ à $1,2 \mathrm{~m}$ puis décroît jusqu'à $0,450 \mathrm{~mm}$ à $1,65 \mathrm{~m}$. Elle augmente considérablement à $1 \mathrm{~mm}$ à $1,82 \mathrm{~m}$ puis baisse progressivement jusqu'à 0,440 $\mathrm{mm}$ à 2,8 $\mathrm{m}$. De là, elle augmente à $0,740 \mathrm{~mm}$ à $3 \mathrm{~m}$ avant de décroître en dents de scie jusqu'à atteindre $0,050 \mathrm{~mm}$ à $3,68 \mathrm{~m}$. Elle augmente à nouveau et progressivement jusqu'à atteindre $0,410 \mathrm{~mm}$ à $4,08 \mathrm{~m}$ avant de baisser 0,050 $\mathrm{mm}$ à $4,8 \mathrm{~m}$ au sommet de l'unité 4 . Ensuite, elle augmente à nouveau à 0,410 $\mathrm{mm}$ à $5,05 \mathrm{~m}$ et oscille en dents de scie entre $0,440 \mathrm{~mm}$ et $0,050 \mathrm{~mm}$ de 5,05 $\mathrm{m}$ à $6,7 \mathrm{~m}$. Enfin, elle augmente ensuite progressivement jusqu'à atteindre à $0,530 \mathrm{~mm}$ à $7,7 \mathrm{~m}$ puis baisse par la suite progressivement à $0,411 \mathrm{~mm}$ à 8,55 m et augmente à $0,440 \mathrm{~mm}$ à 9,1 $\mathrm{m}$ au sommet du profil ;

le mode augmente d'abord de $0,125 \mathrm{~mm}$ à $0,250 \mathrm{~mm}$ entre $0,7 \mathrm{~m}$ et 1,2 $\mathrm{m}$, puis baisse à $0,125 \mathrm{~mm}$ à $1,65 \mathrm{~m}$. Il augmente brutalement à $2 \mathrm{~mm}$ à 1,85 $\mathrm{m}$ et chute considérablement à $0,125 \mathrm{~mm}$ de $2,2 \mathrm{~m}$ à $2,8 \mathrm{~m}$. Il augmente à nouveau $0,250 \mathrm{~mm}$ à $3 \mathrm{~m}$, puis baisse jusqu'à atteindre $0,050 \mathrm{~mm}$ à 3,68 $\mathrm{m}$. Il augmente encore à $0,125 \mathrm{~mm}$ à $4,08 \mathrm{~m}$ puis baisse à $0,050 \mathrm{~mm}$ à $4,08 \mathrm{~m}$ au sommet de l'unité 4. Ensuite, le mode augmente et oscille en dents de scie entre $0,250 \mathrm{~mm}$ et $0,050 \mathrm{~mm}$ de $5,05 \mathrm{~m}$ à $6,7 \mathrm{~m}$ puis augmente progressivement jusqu'à atteindre $0,250 \mathrm{~mm}$ à $7,4 \mathrm{~m}$, ensuite il diminue à $0,125 \mathrm{~mm}$ et y reste constant jusqu'au sommet du profil ;

la moyenne quant à elle augmente de $0,225 \mathrm{~mm}$ à $0,265 \mathrm{~mm}$ de $0,7 \mathrm{~m}$ à $1,2 \mathrm{~m}$, puis baisse à $0,208 \mathrm{~mm}$ à $1,65 \mathrm{~m}$. Elle augmente considérablement à $0,456 \mathrm{~mm}$ à $1,85 \mathrm{~m}$ puis chute à $0,223 \mathrm{~mm}$ à $2,8 \mathrm{~m}$. Elle augmente à nouveau à $0,297 \mathrm{~mm}$ à $3 \mathrm{~m}$ puis baisse à $0,050 \mathrm{~mm}$ à $3,68 \mathrm{~m}$. Elle augmente progressivement jusqu'à $0,195 \mathrm{~mm}$ à $4,08 \mathrm{~m}$ avant de baisser à $0,050 \mathrm{~mm}$ à 4,8 $\mathrm{m}$ au sommet de l'unité 4. Ensuite, la moyenne augmente et oscille en dents de scie entre $0,239 \mathrm{~mm}$ et $0,050 \mathrm{~mm}$ de $5,05 \mathrm{~m}$ à $6,7 \mathrm{~m}$. De là, la moyenne augmente à nouveau, progressivement jusqu'à 0,259 mm à 7,4 mm puis baisse jusqu'à $0,189 \mathrm{~mm}$ à $8,3 \mathrm{~m}$ et enfin augmente légèrement à 0,202 mm à 9,1 $\mathrm{m}$ au sommet du profil ;

l'écart-type croît de 0,69 à 0,80 de $0,7 \mathrm{~m} \mathrm{1,35} \mathrm{m}$ puis baisse à 0,720 à $1,65 \mathrm{~m}$. Il augmente fortement à 1,055 à $1,85 \mathrm{~m}$ avant de baisser progressivement à 0,569 à $2,6 \mathrm{~m}$. Il augmente à nouveau jusqu'à 0,772 à $3 \mathrm{~m}$ puis baisse en dents de scie à 0,40 à $3,68 \mathrm{~m}$. L'écart-type augmente encore à 0,641 à $4,08 \mathrm{~m}$ puis baisse encore jusqu'à 0,421 à $4,8 \mathrm{~m}$ au sommet de l'unité 4. Ensuite, il augmente à 0,719 à 5,05 m et oscille entre 0,719 et 0,399 de 5,07 $\mathrm{m}$ à $6,7 \mathrm{~m}$. De là, l'écart-type augmente à 0,696 à $7 \mathrm{~m}$ puis croit légèrement jusqu'à atteindre 0,754 à 9,1 au sommet du profil ;

l'asymétrie croit d'abord de $-0,019$ à 0,172 de $0,7 \mathrm{~m}$ à $1,2 \mathrm{~m}$ puis diminue à $-0,020$ ? à $1,65 \mathrm{~m}$. Elle augmente à nouveau à 0,250 à $1,85 \mathrm{~m}$ avant de diminuer progressivement jusqu'à $-0,144$ à 2,6 m. Elle augmente encore à 0,075 à $3 \mathrm{~m}$, reste constante à 0,020 à 3,6 m puis augmente encore brutalement 
à 0,321 à $6,68 \mathrm{~m}$ avant de baisser jusqu'à $-0,045$ à $4,08 \mathrm{~m}$. Elle augmente à nouveau 0,350 à $4,8 \mathrm{~m}$ au sommet de l'unité 4 . L'asymétrie chute à 0,018 à $5,05 \mathrm{~m}$ à la base de l'unité 5 puis augmente d'abord en dents de scie entre 0,018 et 0,333 de $5,05 \mathrm{~m}$ à $5,3 \mathrm{~m}$ et ensuite considérablement à 2,160 à 5,95 $\mathrm{m}$ avant de chuter à 0,022 à $6,15 \mathrm{~m}$. Par la suite, elle oscille entre 0,022 et 0,306 de $6,15 \mathrm{~m}$ à $6,7 \mathrm{~m}$. De $6,7 \mathrm{~m}$ au sommet du profil, l'asymétrie varie très peu entre 0,115 et $-0,015$.

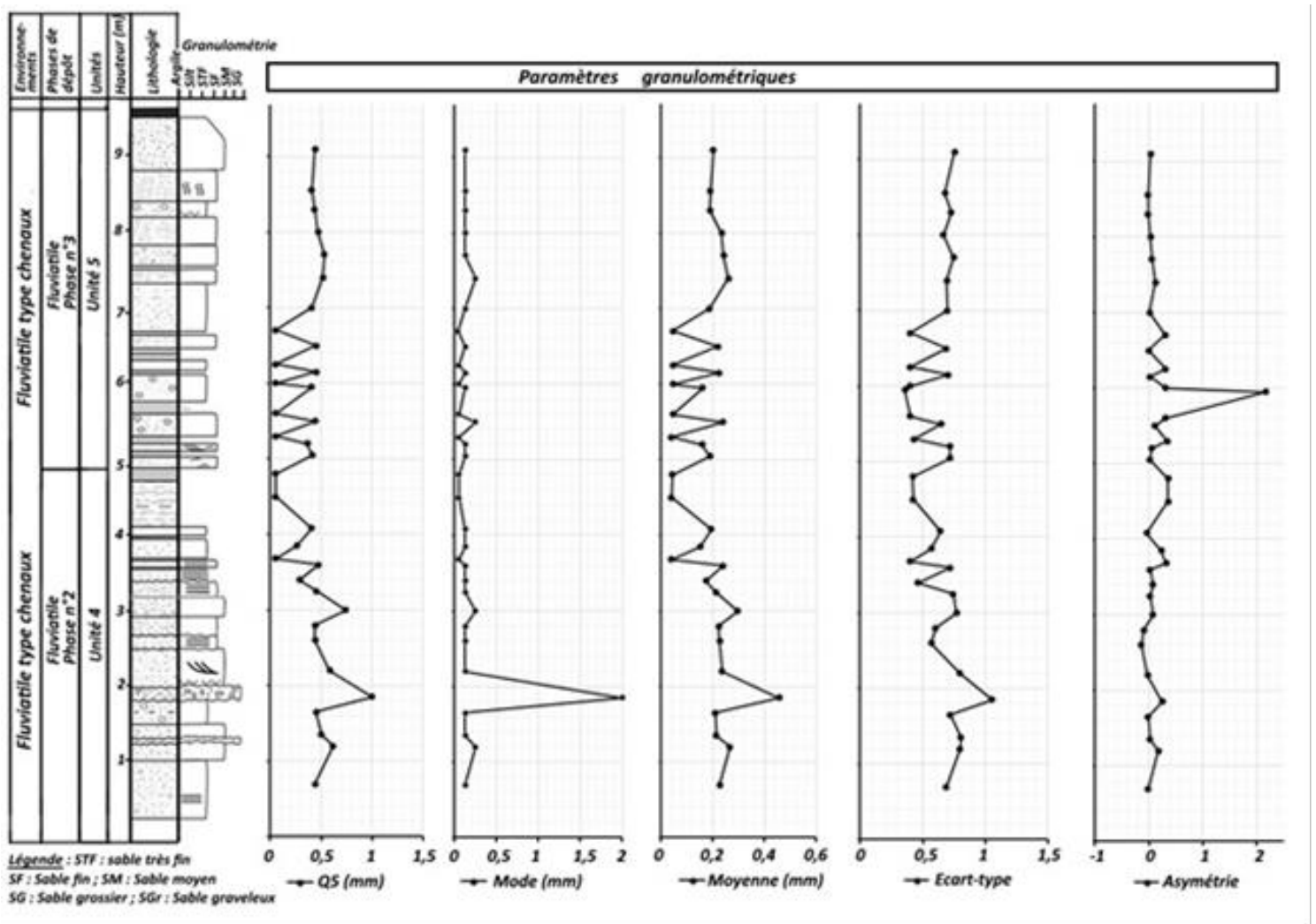

Figure 8. Variations des paramètres granulométriques le long du profil de Kivesso 8

L'évolution des paramètres granulométriques le long du profil de KVS8 confirme les deux unités mises en évidence par l'étude sur le terrain. Elle met en évidence une unité 4 marquée dans sa partie inférieure par une moyenne et une valeur de Q5 élevées et qui diminuent progressivement au sommet de l'unité, alors que l'unité 5 est caractérisée d'abord par une évolution en dents de scie de la moyenne et de Q5, et ensuite par une augmentation progressive de la moyenne et de Q5 qui diminuent par la suite progressivement jusqu'au sommet de l'unité 5 . Ceci correspond à un environnement sédimentaire à hydrodynamisme variable.

Dans le profil de Kivesso 4 (Figure 9), l'évolution des paramètres granulométriques montre du bas vers le haut que : 
- $\quad$ la valeur du percentile Q5 baisse d'abord considérablement de 0,67 $\mathrm{mm}$ à $0,050 \mathrm{~mm}$ de $0,7 \mathrm{~m}$ à $1,2 \mathrm{~m}$, puis elle évolue en dents de scie entre 0,483 $\mathrm{mm}$ et $0,040 \mathrm{~mm}$ de $1,5 \mathrm{~m}$ à $3,15 \mathrm{~m}$ au sommet de l'unité 3 . À partir de 3,15 $\mathrm{m}$, elle augmente progressivement jusqu'à atteindre $0,637 \mathrm{~mm}$ à $4,4 \mathrm{~m}$, puis chute à $0,126 \mathrm{~mm}$ au sommet de l'unité 4 . Enfin, la valeur de Q5 augmente à $0,595 \mathrm{~mm}$ au sommet du profil à $5 \mathrm{~m}$;

- $\quad$ le mode baisse d'abord considérablement de $0,200 \mathrm{~mm}$ à $0,050 \mathrm{~mm}$ de $0,7 \mathrm{~m}$ à $1,63 \mathrm{~m}$ puis évolue ensuite en dents de scie entre $0,200 \mathrm{~mm}$ et 0,050 $\mathrm{mm}$ de $1,63 \mathrm{~m}$ à $3,15 \mathrm{~m}$ au sommet de l'unité 3 . Il augmente à nouveau à 0,200 $\mathrm{mm}$ à $3,6 \mathrm{~m}$, restant constant jusqu'à $4,78 \mathrm{~m}$ puis baisse à $0,125 \mathrm{~mm}$ à $4,85 \mathrm{~m}$ au sommet de l'unité 4 , avant d'augmenter à $0,200 \mathrm{~mm}$ à $5 \mathrm{~m}$, au sommet du profil ;

- $\quad$ la moyenne de même baisse d'abord considérablement de $0,435 \mathrm{~mm}$ à $0,050 \mathrm{~mm}$ de $0,7 \mathrm{~m}$ à $1,2 \mathrm{~m}$, puis, augmente à $0,186 \mathrm{~mm}$ à $1,5 \mathrm{~m}$. Ensuite, elle évolue en dents de scie entre $0,218 \mathrm{~mm}$ et $0,120 \mathrm{~mm}$ de $1,5 \mathrm{~m}$ à $3,15 \mathrm{~m}$ au sommet de l'unité 3. Ensuite, la moyenne augmente progressivement de la base de l'unité 4 à $3,15 \mathrm{~m}$ à $4,4 \mathrm{~m}$ où elle atteint $0,282 \mathrm{~mm}$ puis elle diminue en dents de scie jusqu'à $0,158 \mathrm{~mm}$ à $4,85 \mathrm{~m}$ au sommet de l'unité 4 . Enfin, elle augmente à $0,291 \mathrm{~mm}$ à $5 \mathrm{~m}$, au sommet du profil ;

- $\quad$ l'écart-type augmente d'abord de 0,761 à 0,950 de $0,7 \mathrm{~m}$ à $1,63 \mathrm{~m}$, puis diminue à 0,80 à $1,8 \mathrm{~m}$. Il augmente à nouveau à 0,894 à $1,88 \mathrm{~m}$ et baisse progressivement à 0,80 à $2,13 \mathrm{~m}$. Ensuite, il évolue en dents de scie entre 0,883 et 0,880 de $2,13 \mathrm{~m}$ à $3,15 \mathrm{~m}$ au sommet de l'unité 3 . De là, l'écart-type baisse progressivement de la base de l'unité 4 jusqu'à $4,4 \mathrm{~m}$ où il atteint 0,755 . Ensuite, il augmente à 0,823 à $4,6 \mathrm{~m}$ puis baisse à 0,734 à 4,78 avant d'augmenter à 0,999 à $4,85 \mathrm{~m}$ au sommet de l'unité 4 . Enfin, l'écart-type baisse à 0,707 à $5 \mathrm{~m}$, au sommet du profil ; 


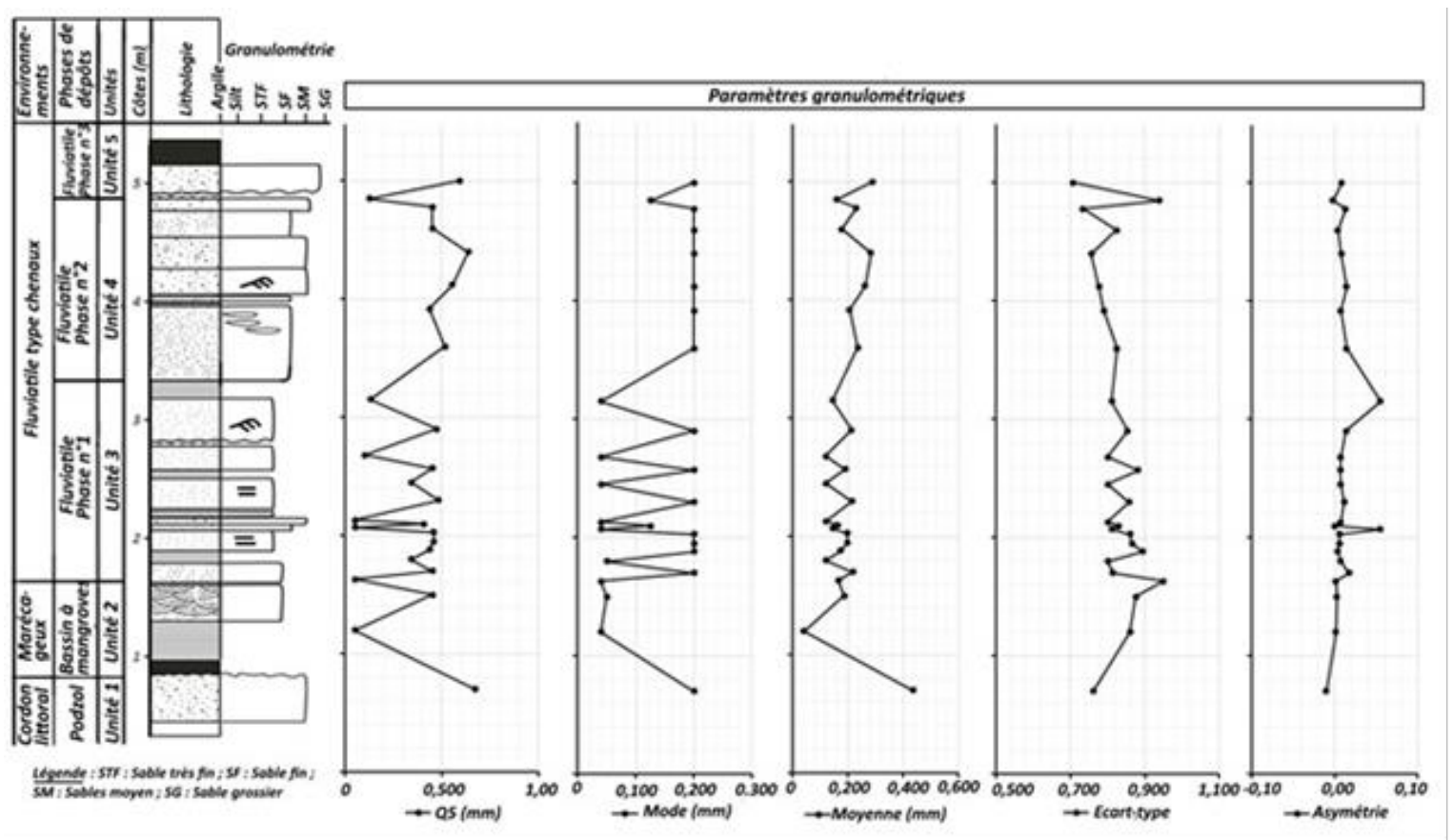

Figure 9. Variations des paramètres granulométriques le long du profil de Kivesso 4

- l'asymétrie augmente d'abord légèrement de $-0,108$ à 0,023 de $0,7 \mathrm{~m}$ à $1,63 \mathrm{~m}$, puis considérablement à 0,168 à $1,7 \mathrm{~m}$ avant de baisser progressivement à 0,035 à $1,88 \mathrm{~m}$. Elle augmente à nouveau considérablement à 0,550 à $2,07 \mathrm{~m}$ puis chute à $-0,006$ à $2,1 \mathrm{~m}$ avant d'augmenter légèrement et progressivement jusqu'à atteindre 0,070 à $2,68 \mathrm{~m}$, puis brutalement à 0,550 à $3,15 \mathrm{~m}$ au sommet de l'unité 3 . De là, elle diminue progressivement jusqu'à atteindre - 0,021 à $4,85 \mathrm{~m}$, au sommet de l'unité 4 . Enfin, elle augmente à 0,082 à $5 \mathrm{~m}$, au sommet du profil.

- $\quad$ L'évolution des paramètres granulométriques le long du profil de KVS 4 confirme les cinq unités mises en évidence par l'étude de terrain. Elle met en évidence une unité 1 marquée par une valeur élevée de la moyenne et du percentile Q5, alors que les unités 2 à 3 qui la surmontent sont marquées par une évolution en dent de scie de la moyenne et du Q5. L'unité 4 montre d'abord une augmentation progressive de la moyenne et de Q5 dans ses parties inférieure et moyenne, ensuite une baisse progressive des valeurs de ces deux paramètres dans sa partie supérieure.

\subsubsection{Mode de transport et conditions de sédimentation}

Le diagramme de C-M de Passega (1957) (Figure 10), qui permet de déterminer les conditions de transport, situe les sables de Kivesso, majoritairement, dans le segment R-Q qui correspond au mode de transport par suspension graduée sans roulement; c'est-à-dire, par saltation et 
secondairement dans le domaine $\mathrm{T}$ qui correspond au mode de transport par suspension pélagique.

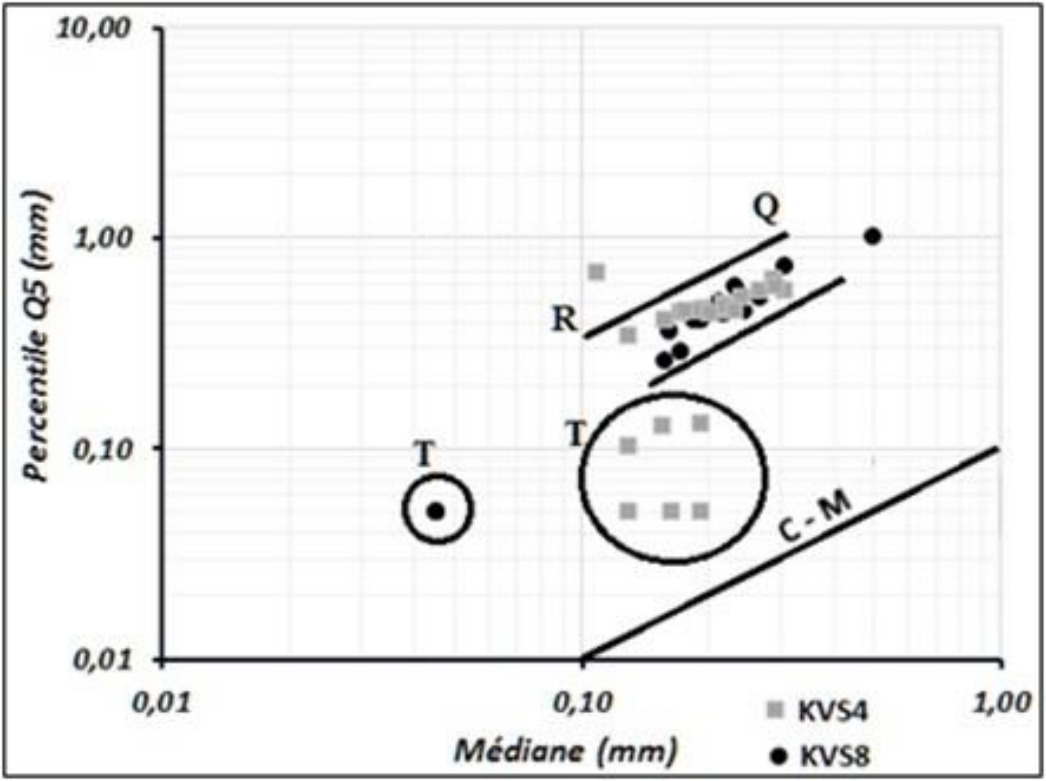

Figure 10. Diagramme C-M de Passega des sables de Kivesso

Le diagramme de Losson et al. (2006) (Figure 11) qui permet de déterminer le mode de sédimentation, situe les sédiments dans le domaine 4 qui correspond à la sédimentation par accumulation libre. Les sédiments remplissant la baie de Loango dans le secteur de Kivesso ont donc été abandonnés progressivement par simple gravité au fur et à mesure que le courant transporteur perdait sa compétence. 


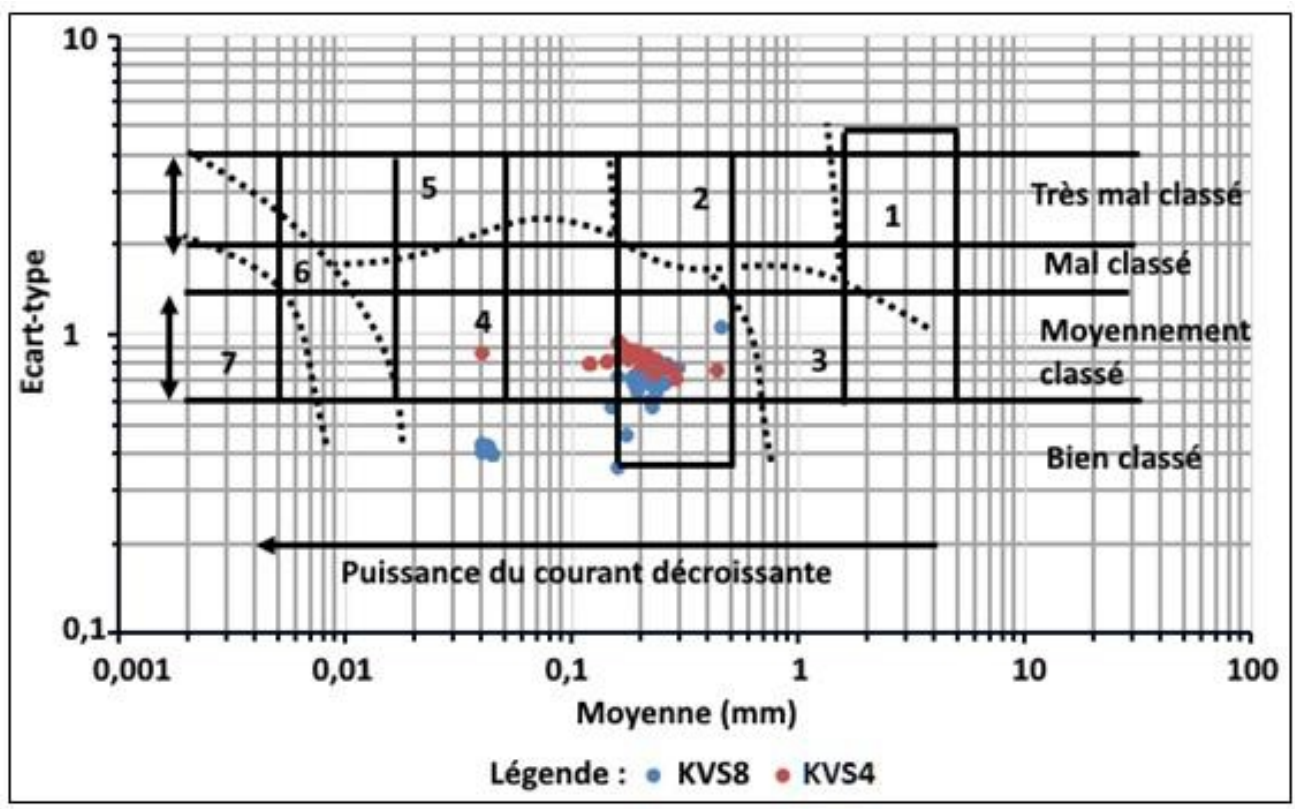

Figure 11. Diagramme de Losson des sables de Kivesso

Légende : (1). Accumulation forcée ; (2). Accumulation forcée puis libre ; (3). Accumulation libre proche du dépôt forcé ; (4). Accumulation libre ; (5). Dépôt en vrac ; (6). Transition; (7). Décantation

\subsubsection{Morphoscopie et minéralogie}

L'observation à la loupe binoculaire (Figure 12) montre des sables essentiellement quartzeux à plus de $95 \%$. Les quartz ronds et sub arrondis mats représentent environ $60 \%$, ils tendent à devenir progressivement luisants (Figure $\left.12 \mathrm{a}, \mathrm{n}^{\circ} 1-2\right)$. Ils sont associés aux grains de quartz ronds et sub arrondis luisants (environ $35 \%$ ) alors que les débris lithiques (quartzites, latérite, grès siliceux) représentent environ $4 \%$ (Figure 12a, $\mathrm{n}^{\circ} 3-5$ ). Les minéraux lourds sont peu abondants. Certains quartz ont des inclusions de minéraux noirs bien cristallisés. Les quartz montrent couramment des marques de chocs bien polies représentées par les croissants de choc, le V, les cupules, les cassures, les figures de stress dues aux chocs et les fissures (Figure 12b, $\mathrm{n}^{\circ} 1-3$ ). 

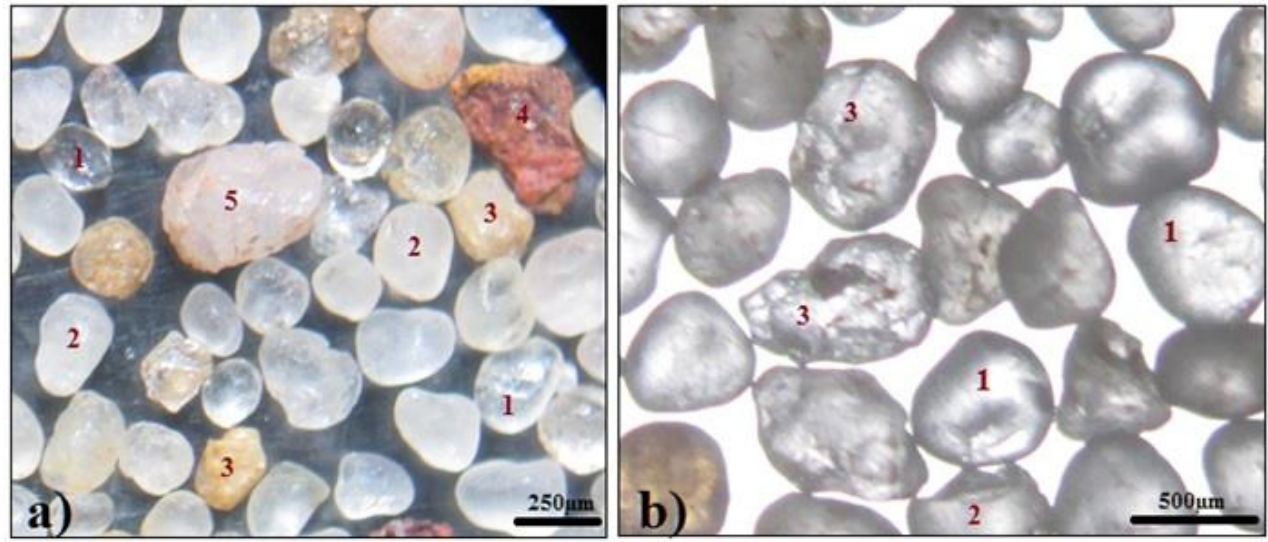

Figure 12. Morphoscopie des sables Holocène de la baie de Loango, secteur de Kivesso

Photo a) : 1. Grain de quartz luisant et cassé ; 2. Grain de quartz rond mat; 3. Débris de quartzite; 4. Débris non usé de latérite; 5 . Débris de silice recristallisée. Photo b) : $\mathbf{1}$. Grain de quartz portant un impact de choc ; 2 . Grain de quartz rond cassé ; 3. Grain de quartz fortement dissout et enduit d'oxyde de fer sur les traces de dissolution.

Ces marques de choc sont fréquemment tapissées ou remplies d'un enduit rouge ocre d'oxyde de fer (Figure 12b, $\mathbf{n}^{\circ} \mathbf{3}$ ). Les traces de dissolution sont fréquentes et représentées par les carries et les rigoles parfois anastomosées (Figure 12b, $\mathbf{n}^{\circ} \mathbf{3}$ ). Quelques rares quartz montrent des figures de dissolution triédriques. Ces figures de dissolution se superposent parfois sur les marques de chocs. Elles sont parfois tapissées d'un enduit d'oxyde de fer ou parfois portent des amas de silice microcristallines ou amorphes. Certains quartz ont des surfaces tapissées de microcristaux de silice. Les figures de corrosion ainsi que les encroutements d'oxyde de fer sont également observées sur les quartz. Les débris lithiques sont les débris de quartzite arrondis et anguleux, ceux de latérite sont généralement usés et constitués de cuirasse latéritique et de latérite à aspect bréchique à grains de quartz essentiellement luisants liés par le fer (Figure 12a, $\mathbf{n}^{\circ} \mathbf{3}-4$ ). Les agrégats sont de deux types : les agrégats à fins quartz à ciment siliceux et ceux à ciment ferrugineux. On observe aussi de temps en temps des fleurs de silice, des quartz recristallisés. Les minéraux lourds sont peu abondants, il s'agit par ordre décroissant de l'ilménite, la magnétite, le rutile, le zircon, la tourmaline, le sphène, le disthène, l'amphibole brune, le grenat. Ces minéraux sont majoritairement usés. Ces caractéristiques nous les avons aussi observées dans les sédiments silico-clastiques de la série des Gorges.

\subsubsection{Minéralogie des argiles}

Les analyses RX effectuées sur les échantillons de la partie basale du profil de KVS 4 (Tableau 2) montrent que la minéralogie des argiles est dominée au moins à $90 \%$ par la kaolinite associée à l'halloysite (1 à $6 \%$ ). 
L'illite est présente mais en proportion très faible, inférieure ou égale à $4 \%$. Par contre, la Montmorillonite et la smectite sont en trace dans le bassin marécageux et sont associées à de traces de feldspath. Verticalement (Tableau 1), la proportion en halloysite évolue de manière inversement proportionnelle à celle de la kaolinite (Figure 13). Par contre, celles obtenues dans le profil de Kivesso 2, qui correspond stratigraphiquement à la partie supérieure du profil de Kivesso 4, montrent une dominance totale de la kaolinite. L'halloysite est presque absente et n'apparaît qu'en proportion erratique en un seul point, alors que les argiles de type 2/1 disparaissent complètement.

Tableau 2. Proportions des minéraux argileux présents dans la partie inférieure de KVS4 et la partie supérieure de KVS 2

\begin{tabular}{|l|l|l|l|l|l|l|}
\hline Echantillons & Hauteurs & $\begin{array}{l}\text { Illite } \\
(\%)\end{array}$ & $\begin{array}{l}\text { Kaolinite } \\
(\%)\end{array}$ & $\begin{array}{l}\text { Halloysite } \\
(\%)\end{array}$ & $\begin{array}{l}\text { Montmorillonite } \\
(\%)\end{array}$ & $\begin{array}{l}\text { Smectite } \\
(\%)\end{array}$ \\
\hline KVS $4-2$ & +1.20 & 0,00 & 86,50 & 0,00 & 0,00 & 13,5 \\
KVS $4-3$ & $+1,50$ & 3,50 & 57,50 & 39,00 & 0,00 & 0,00 \\
KVS $4-4$ & $+1,63$ & 4,00 & $96 ? 00$ & 0,00 & 0,00 & 0,00 \\
KVS $4-7$ & $+1,88$ & 5,00 & 87,50 & 7,50 & 0,00 & 0,00 \\
KVS $4-8$ & $+1,95$ & 3,00 & 94,50 & 0,00 & 2,50 & 0,00 \\
KVS $4-9$ & $+2,03$ & 3,00 & 97,00 & 0,00 & 0,00 & 0,00 \\
KVS $4-11$ & $+2,10$ & 3,00 & 86,00 & 11,00 & 0,00 & 0,00 \\
KVS $4-13$ & $+2,30$ & 3,50 & 92,50 & 4,00 & 0,00 & 0,00 \\
KVS $4-14$ & $+2,45$ & 0,00 & 97,50 & 0,00 & 0,00 & 0,00 \\
KVS $4-15$ & $+2,57$ & 3,00 & 97,00 & 0,00 & 0,00 & 0,00 \\
KVS $4-17$ a & $+2,90$ & 6,00 & 94,00 & 0,00 & 0,00 & 0,00 \\
KVS $4-17 b$ & $+3,00$ & 4,00 & 73,00 & 20,00 & 0,00 & 3,00 \\
KVS $2-17$ & $+3,20$ & 2,70 & 94,50 & 0,00 & 13,62 & 0,00 \\
KVS $2-18$ & $+3,40$ & 3,50 & 95,50 & 0,00 & 0,00 & 0,00 \\
KVS $2-20$ & $+3,60$ & 2,50 & 89,50 & 8,00 & 0,00 & 0,00 \\
KVS $2-21$ & $+3,70$ & 0,00 & 100,00 & 0,00 & 0,00 & 0,00 \\
KVS 2 - 22 & $+3,80$ & 1,50 & 98,500 & 0,00 & 0,00 & 0,00 \\
\hline
\end{tabular}




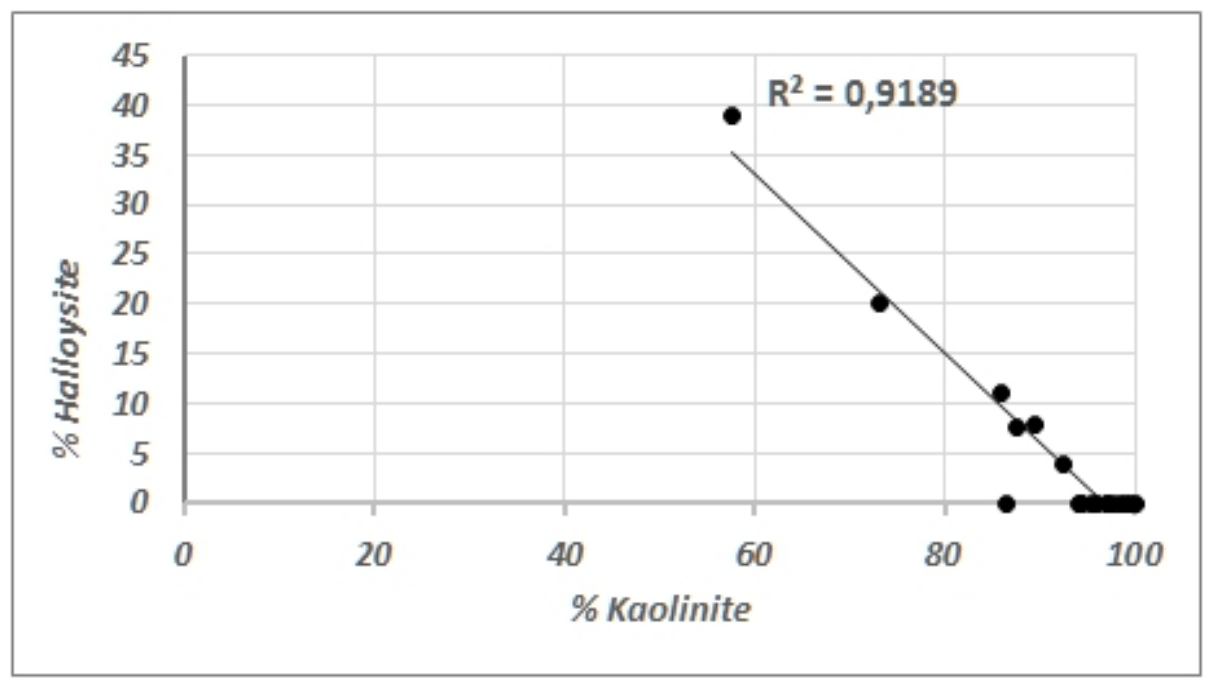

Figure 13. Relation entre l'halloysite et la kaolinite dans la partie inférieure de KVS4 et dans la partie supérieure de KVS2

\subsubsection{Analyses chimiques}

Les teneurs en carbone organique, en azote total et en matière organique (MO) obtenues le long du profil de KVS 4 (Tableau 3) sont très faibles excepté dans les échantillons situés dans le bassin marécageux. Elles varient entre 0,03 et $17,02 \%$ pour le carbone ; entre 0,01 et $0,59 \%$ pour l'azote ; entre 0,04 et $29,34 \%$ pour la matière organique, alors que le rapport $\mathrm{C} / \mathrm{N}$ est inférieur à 10 sauf à $1,32 \mathrm{~m}$ et $2,7 \mathrm{~m}$ où la valeur de ce rapport atteint respectivement $29,34 \%, 8,58 \%$ suggérant ainsi une matière organique essentiellement moins mature. 
Tableau 3. Teneur en carbone organique, en azote total, en matière organique et la valeur $d u$ rapport $C / N$ dans les échantillons de Kivesso 4

\begin{tabular}{|l|l|l|l|l|l|}
\hline Echantillons & Hauteurs $(\boldsymbol{m})$ & $\boldsymbol{C}(\boldsymbol{\%})$ & $\boldsymbol{M O}(\boldsymbol{\%})$ & $\boldsymbol{N}(\boldsymbol{\%})$ & $\boldsymbol{C} / \boldsymbol{N}$ \\
\hline KVS $4-1$ & $+1,00$ & 0,05 & 0,08 & 0,04 & 1,16 \\
KVS 4 - & $+1,32$ & $\mathbf{1 7 , 0 2}$ & $\mathbf{2 9 , 3 4}$ & 0,59 & $\mathbf{2 8 , 8 5}$ \\
KVS $4-3$ & $+1,62$ & 0,07 & 0,12 & 0,02 & 3,48 \\
KVS 4 - 5 & $+1,64$ & 0,14 & 0,24 & 0,02 & 6,96 \\
KVS 4 - 7 & $+2,02$ & 0,05 & 0,09 & 0,03 & 1,74 \\
KVS 4 - 8 & $+2,07$ & 0,08 & 0,13 & 0,03 & 2,51 \\
KVS 4 - & $+2,12$ & 0,05 & 0,08 & 0,06 & 0,77 \\
KVS 4 - 11 & $+2,15$ & 0,03 & 0,06 & 0,24 & 0,15 \\
KVS 4 - 13 & $+2,40$ & 0,06 & 0,10 & 0,03 & 1,93 \\
KVS 4 - 14 & $+2,47$ & 0,29 & 0,50 & 0,12 & 2,42 \\
KVS 4 - 15 & $+2,66$ & 0,10 & 0,18 & 0,02 & 5,22 \\
KVS 4 - 16 a & $+2,70$ & $\mathbf{4 , 9 8}$ & $\mathbf{8 , 5 8}$ & 0,04 & $\mathbf{1 2 4 , 4 2}$ \\
KVS 4 - 17 a & $+2,90$ & 0,07 & 0,12 & 0,02 & 3,48 \\
KVS 4 - 17 b & $+3,04$ & 0,06 & 0,10 & 0,02 & 2,90 \\
KVS 4 - 18 & $+3,24$ & 0,54 & 0,93 & 0,06 & 8,99 \\
KVS 4 - 19 a & $+3,54$ & 0,10 & 0,18 & 0,03 & 3,48 \\
KVS 4 - 19 b & $+3,79$ & 0,02 & 0,04 & 0,03 & 0,77 \\
KVS 4 - 20 & $+3,87$ & 0,08 & 0,14 & 0,04 & 2,03 \\
KVS 4 - 21 & $+3,97$ & 0,06 & 0,11 & 0,02 & 3,19 \\
KVS 4 - 21 b & $+4,10$ & 0,07 & 0,12 & 0,03 & 2,32 \\
KVS 4 - 22 & $+4,36$ & 0,04 & 0,07 & 0,03 & 1,35 \\
KVS 4 - 23 & $+4,58$ & 0,17 & 0,29 & 0,03 & 5,61 \\
KVS 4 - 24 & $+4,68$ & 0,14 & 0,24 & 0,03 & 4,64 \\
KVS 4 - 25 & $+4,72$ & 0,31 & 0,54 & 0,04 & $\mathbf{7 , 8 3}$ \\
KVS 4 - 26 & $+4,96$ & 0,09 & 0,15 & 0,01 & $\mathbf{8 , 7 0}$ \\
\hline
\end{tabular}

D'une manière générale, les teneurs en carbone et en matière organique, légèrement élevées dans la partie basale du profil, diminuent vers le sommet puis augmentent légèrement à nouveau dans le dernier mètre avec un enrichissement à partir de 4,72 m. Cet enrichissement en matière organique au sommet du profil est dû à la décomposition du couvert végétal actuel.

\subsubsection{Phases de sédimentation et datations au radiocarbone $\left({ }^{14} \mathrm{C}\right)$}

Les différents âges obtenus des datations au radiocarbone $\left({ }^{14} \mathrm{C}\right)$ à partir des débris végétaux provenant des profils de Kivesso (Figure 14) indiquent des sédiments relativement jeunes, dont les âges varient entre 500 ans B.P. et au moins 100 ans B.P. La répartition verticale de ces âges le long des profils, met en évidence une zone marécageuse peu profonde qui couvrait tout le secteur de Kivesso. Cette zone s'est mise en place sur un cordon sableux littoral, érigé lors de la dernière régression holocène. Elle a fonctionné entre 500 ans B.P. et 300 ans B.P. Puis, elle a progressivement été colmatée par des sédiments silico-clastiques provenant de l'érosion des formations de la série des Gorges et transportés par des cours d'eau à compétence contrastée. La 
première phase de colmatage s'est faite entre 500 ans à 350 ans B.P., et elle correspond aux sables moyen et fin, beige et blanchâtre ; la deuxième entre 350 ans à 223 ans B.P., correspond au sable rouge ocre à niveaux conglomératiques et graveleux, enfin la troisième phase de 223 ans B.P. à environ 100 ans B.P., corresponde au sable moyen, beige et blanc localement graveleux ou conglomératique à la base. Les paléosols végétaux datés à 350 ans B.P. à Kivesso 4 ; 223 ans B.P. et 100 ans B.P. à Kivesso 2 suggèrent des émersions et des arrêts plus ou moins longs de la sédimentation qui étaient localement accompagnés par des érosions d'ampleur variable. Les sédiments datés à 7000 ans B.P. n'ont été rencontrés qu'à KVS7.

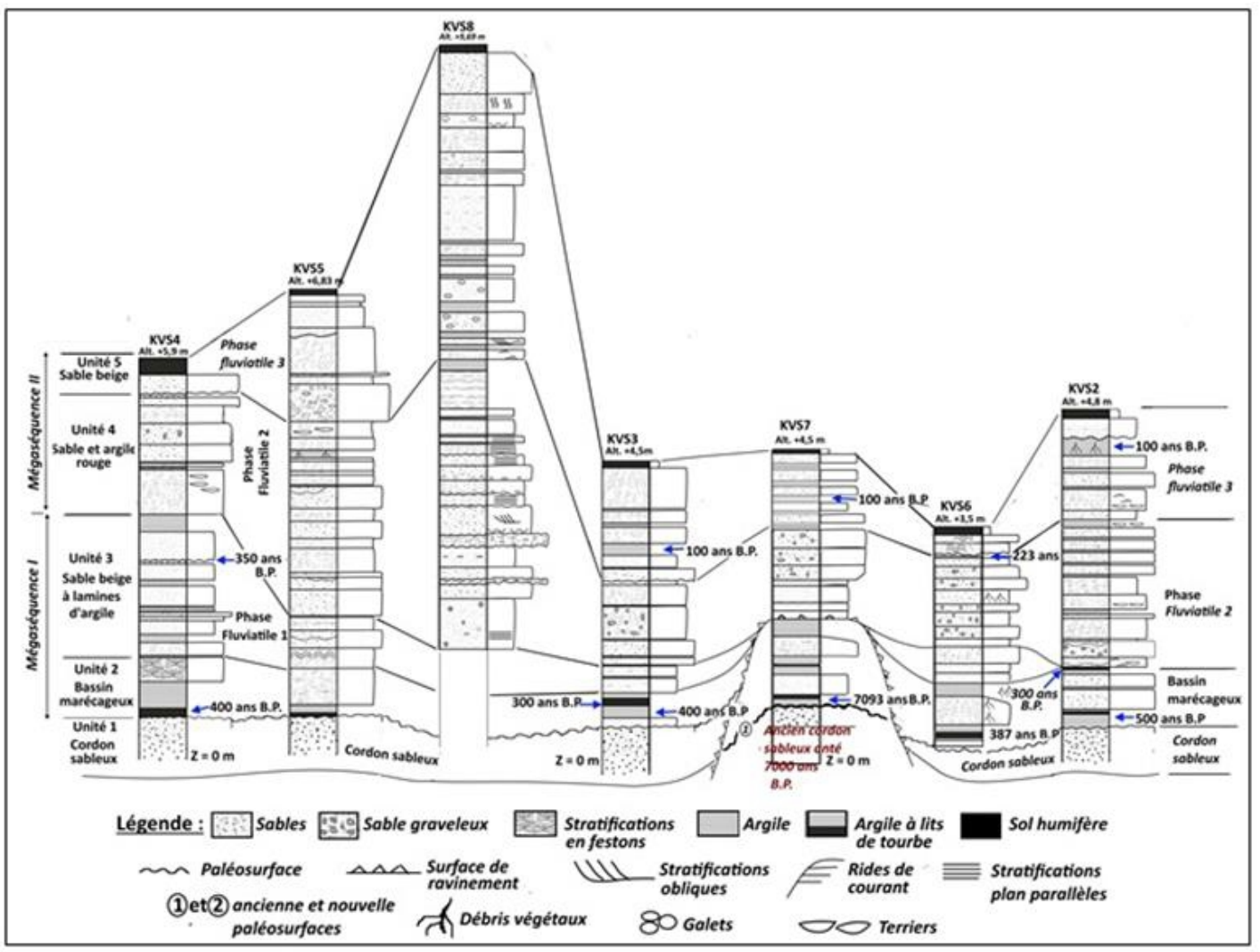

Figure 14. Corrélation entre les différents logs lithologiques de Kivesso

\section{Interprétation}

La description des différents faciès lithologiques montre que le remplissage sédimentaire de la baie de Loango dans le secteur de Kivesso est essentiellement silico-clastique, structuré en cinq unités lithologiques qui correspondent à trois environnements sédimentaires. La première unité (unité 1) correspond au cordon sableux littoral ancien sur lequel se développe l'unité 2 qui correspond à la zone marécageuse et les trois autres correspondent à l'environnement fluviatile. Le cordon sableux littoral, s'est mis en place lors 
de la dernière régression Holocène et a subi une podzolisation. En effet, Giresse et Kouyoumontzakis (1974) distinguent le long du littoral congolais trois cordons sableux étagés qui auraient subi plusieurs phases de podzolisation (Schwartz, 1990).

L'unité 2 constituée à la base par de l'argile plastique, marron à intercalations de niveaux de tourbe contenant des débris de tiges, d'écorces et de racines suggère l'existence d'une zone marécageuse dans laquelle s'est développée une forêt ombrophile. Les alternances interstratifiées d'argile avec les lamines de matière organique, suggèrent un climat humide a saisons contrastées.

L'unité 4 constituée à KVS 8 de sables moyens et grossiers à intercalations de petits niveaux de terrasse alluviale, à stratifications plans parallèles, à stratification entrecroisées marquées par des granules devenant de plus en plus fins vers le haut, révèlent la présence d'une terrasse constituée de graviers aplatis de quartzite et de latérite, de blocs roulés d'argilite rouge et de blocs anguleux de latérite emballés dans une matrice sablo-argileuse. Son évolution latérale à KVS 4 par des sables moyens à fins, rouge ocre renferme des galets mous d'argilite rouge et intercalés de lits d'argile rouge ocre contenant parfois des débris de végétaux. Les nombreuses gouttières d'érosion remplies par du sable blanc à stratifications obliques progradantes tantôt vers le sud tantôt vers le nord, à rides de courant observées dans cette unité permettent d'envisager une reprise de l'action érosive des cours d'eau de leurs propres chenaux. Dans sa partie supérieure, cette unité constituée de sables fins à moyen, à rides de courant, à stratifications obliques associées à des stratifications plan parallèles et qui se terminent par des lits d'argile sableuse parfois contenant de la matière organique suggèrent le retour des cours d'eau à leur dynamique habituelle tels que décrite dans l'unité 3 .

L'unité 5 constituée par des sables fins et moyens, blanc et jaune ocre, assez-bien à moyennement classés, à intercalations de lits d'argile grise, confirme le système fluviatile méandriforme qui marque la fin du colmatage des chenaux dans notre secteur d'étude, qui est resté longtemps émergé et soumis à des multiples érosions; d'où l'existence que de sa partie basale dans la majeure partie du secteur de Kivesso, à l'exception de KVS 8 où l'unité 5 est plus développée. L'ensemble du dépôt de Kivesso est affecté par une pédogenèse sub actuelle marquée par de nombreuses concrétions ferrifères, les agrégats de fins quartz à ciment ferrugineux et des concrétions latéritiques le long des surfaces de stratification.

La morphoscopie des sables dominés par des quartz usés mats qui tendent à devenir luisants et portant des marques de choc polies, associés aux grains de quartz usés luisants suggère une reprise aquatique des quartz après une éolisation. Les encroutements ferrugineux sur certaines marques de chocs 
ainsi que les traces de dissolution se superposant aux marques de choc suggèrent une immobilisation des quartz après la phase éolienne et un climat tropical humide. Les figures de dissolution de forme triédriques qui se forment selon Rivière (1977) dans la zone intertidale, suggèrent qu'une partie des sables a été apportée par les vents qui balayaient la côte. La silice microcristalline en amas dans les creux de dissolution ou sur la surface des grains de quartz, ainsi que les encroutements ferrifères témoignent de nombreux processus pédogénétiques ayant affectés ces quartz. Les quartz à inclusions noires bien cristallisées, à éclat vitreux témoignent d'une origine magmatique plutonique de certains quartz. La paragenèse de minéraux lourds fortement usés, dominée par l'ilménite, la magnétite, le rutile, le zircon, la tourmaline, le sphène, le disthène, l'amphibole brune et le grenat suggère une origine magmatique et métamorphique. Par contre, la forte usure de ces minéraux implique des remaniements multiples.

Les galets aplatis de quartzite, les granules de quartzite et de latérite, proviennent de l'érosion des formations de la série des Gorges. En effet, Miyouna et al. (2016) et Callec et al. (2015) montrent que la série des Cirques est constituée par des sédiments silico-clastiques structurés en séquences élémentaires constituées par des niveaux alluvionnaires, surmontés par des sables moyens et fins et qui se terminent par des niveaux pélitiques. Les niveaux alluvionnaires contiennent les galets de quartzite, de roches métamorphiques et magmatiques et des granules de latérite. Ces observations sont en accord avec celles de Giresse et Le Ribault (1981) et confirment bien que les sédiments holocènes de la baie de Loango proviennent majoritairement de l'érosion de la série des Cirques et secondairement de la déflation des dunes littorales par les Alysée.

La minéralogie des argiles dominée par la kaolinite, une argile à structure 1/1 qui se forme en milieu continental pourrait en grande partie être héritée de la série des Cirques. En effet, Massengo (1970), Sitou et al. (1992) montrent que la kaolinite est le minérale dominant dans la série des Gorges. L'apparition de l'halloysite uniquement dans la partie inférieure (unités 2 et 3) situé sous les grandes terrasses (unité 4) du dépôt dans le secteur de Kivesso, ainsi que sa relation inverse avec la kaolinite (Figure 12), suggèrent que cette halloysite est autochtone et proviendrait de l'hydratation in-situ de la kaolinite. En conséquence, l'existence de l'halloysite uniquement dans la partie inférieure du dépôt holocène de Kivesso, suggère un léger enfouissement dû à la pression lithostatique ou par jeu de faille. En effet, dans le secteur de Kivesso, les sédiments anciens datés 6 à 7000 ans B.P., rencontrés au sud de la baie de Loango n'ont été rencontrés dans le secteur de Kivesso qu'uniquement à la base de Kivesso 7, au niveau zéro de l'océan actuel. Ceci suggère que ces sédiments anciens ont par endroits été érodés par les houles 
ou soit se retrouvent enfouis en-dessous du niveau marin actuel, par jeu de faille qui aurait développé des horsts et des grabens.

\section{Conclusion}

L'étude sédimentologique, granulométrique et séquentielle associée aux datations au radiocarbone du remplissage sédimentaire Holocène récent de la baie de Loango dans le secteur de Kivesso, met en évidence une zone marécageuse qui couvrait le secteur de Kivesso entre 500 ans et 300 ans B.P. Elle était peu profonde et couverte par une forêt ombrophile. Cette zone marécageuse s'est développée sur un cordon sableux littoral d'origine marine édifié lors de la dernière régression Holocène et ayant subi localement une podzolisation.

Cette zone marécageuse a été progressivement colmatée par des sédiments silico-clastiques provenant majoritairement de l'érosion des formations de la série des Cirques de Diosso et secondairement de la déflation des anciens cordons littoraux par les Alysées. Ces sédiments provenant de la série des Cirques ont été transportés par des cours d'eau à compétence contrastée, parfois torrentielle et se sont déposés par gravité ou par décantation dans un environnement à hydrodynamisme très variable. Le dépôt colmatant la zone marécageuse est structuré en deux méga séquences correspondant à deux phases de colmatage. La première méga séquence dont les âges se situent entre 500 ans B.P. et 300 ans B.P., correspond aux unités 2 et 3. Elle marque le développement des marécages et de leur colmatage par les cours d'eaux côtiers qui prennent naissance aux pieds des Gorges de Diosso et qui coulent sur une plaine côtière basse. Ces cours d'eaux forment un système méandriforme au cours duquel se développent des bancs de sables progradants lors des crues et des lits d'argiles sableuses pendant les décrues. La deuxième méga séquence dont les âges se situent entre 300 ans B.P. et 100 ans B.P., marque le creusement par les cours d'eau de leurs propres chenaux en cherchant de rétablir un nouveau profil d'équilibre au cours duquel se développent des terrasses. Cette deuxième méga séquence se termine par une reprise normale de la dynamique habituelle des cours d'eau tels que décrite dans la première méga séquence.

L'apparition de l'halloysite uniquement dans la première méga séquence (unités 2 et 3 ) situées sous les grandes terrasses (unité 4), suggèrent une hydratation in-situ de la kaolinite héritée de la série des Cirques. Cette évolution de la sédimentation et des paléoenvironnements est en rapport avec la réhumidification du climat à partir de 600 ans B.P. voire 500 ans B.P. tels que décrit par Elenga et al. (1992b et 2001). 


\section{Remerciements}

Nous tenons à remercier Monsieur Jean De-Dieu NZILA, Directeur Général de l'Institut de Recherches en Sciences Exactes et Naturelles (IRSEN), pour la réalisation des analyses granulométriques, texturales et chimiques dans son laboratoire; Monsieur NKODIA Pascal, Directeur Général de Congo Exploration pour l'appui financier et logistique lors des études sur le terrain et de laboratoire. Nos sincères remerciements aux reviewers pour la qualité remarquable de leurs observations qui ont contribué à l'amélioration de ce travail.

\section{References:}

1. Atlas du Congo, ed. J A. (2001). Paris, 14-15p.

2. Bertaux, J., Schwartz, D., Vincens, A., Siffeddine, A., Elenga, H., Mansour, M., Mariotti, A., Fournier, M., Martin, L., Wirrmann, D., \& Servant, M. (2000). Enregistrements de la phase sèche d'Afrique centrale vers 3000 ans BP par la spectrométrie IR dans les lacs Sinnda et Kitina (Sud Congo) Servant $M$ and Servant-Vildary S (eds.), Dynamique à Long Terme des Écosystèmes Forestiers Intertropicaux, IRD/UNESCO/MAB/CNRS, Paris, pp. 43-49.

3. Callec, Y., Lasseur, E., Le Bayon, B., Thiéblemont, T., Fullgraf Gouin, J., Paquet, F., Le Metour, J., Delhaye-Prat, V., Giresse, P., Malounguila-Nganga, D. M., \& Boudzoumou, F. (2015). Notice Explicative de la feuille Pointe Noire à 1/200 000. Programme National de Cartographie Géologique. Ministère des Mines et de la Géologie. Dir. Min. et de la Géol., édition BRGM, 2015, 216 p.

4. DE PLOEY, J. (1963). Quelques indices sur l'évolution morphologique et paléoclimatique des environs du Stanley-Pool (Congo)". Studia Universitatis Lovanium, n¹7, 16 p.

5. Dechamps, R., Guillet, B., \& Schwartz, D. (1988). Découverte d'une flore forestière mi-Holocène (5800-3100 BP) conservée in situ sur le littoral pontenégrin (R.P. du Congo). Compte-Rendus Académie des Sciences, 306 (II) : 615-618.

6. Desthieux, F., Boudzoumou, F., Mompassa, F., Malounguila-Nganga, D. M., Diakubuka, E., Mouanda Moumpassa, R. S., Ondongo, C., Ongouya, A., Akiaoué, E., Missamou, A., Malera, M., Kiba, V., \& Nzaba, M. (1993). Cartographie de la République du Congo à 1/100 000. Ministère des Mines et de l'Energie, Direction Générale des Mines, 1-2, 4-8.

7. Elenga, H. \& Vencens, A. (1990). Paléoenvironnements quaternaires récents des plateaux Batéké (Congo). Etude palynologique des dépôts de la dépression du Bois de Bilanko. In: Lanfranchi R. et Schwartz D. 
Eds., Paysages quaternaires de l'Afrique centrale atlantique. ORSTOM, Paris, 271 - 282.

8. Elenga, H. (1992a). Végétation et climat du Congo depuis 24000 BP. Analyse palynologique de séquences sédimentaires du Pays Batéké et du littoral. Thèse, Université Aix-Marseille, 238 p.

9. Elenga, H., Schwartz, D., Vincens, A. Bertaux, et al. (1996). Diagramme pollinique holocène du lac Kitina (Congo) : mise en évidence de changements paléobotaniques et paléoclimatiques dans le massif forestier du Mayombe. Compte Rendus Acad. Sci., 323 (lia), 403-410.

10. Elenga, H., Schwartz, D., \& Vincens, A. (1992b). Changement climatique et action anthropique sur le littoral congolais au cours de l'Holocène, Bulletin Société Géologique de France, 163, 85-90.

11. Elenga, H., Vincens, A., Schwartz, D., Fabing, D., Berteaux, J., Wirrmann, D., Martin, L., \& Servant, M. (2001). Le marais estuarien de la Songololo (Sud Congo) à l'Holocène moyen et récent. Bulletin Société Géologique de France, 172 (3), 359-366.

12. Folk, R. \& Ward, W. (1957). A study in the significance of grain size parameters. Journal of sedimentary petrology, pp. 3-26

13. Giresse, P. \& Kouyoumontzakis, G. (1974). Observation sur le quaternaire côtier et sous-marins et des régions limitrophes. Aspects eustatiques et climatiques. Association Sénégalaise et Afrique, Bull. liaison, Sénégal, 42-43., pp. 45-61.

14. Giresse, P. \& Lanfranchi, R. (1984). Les climats et les océans de la région congolaise pendant l'Holocène. Bilans selon les échelles et les méthodes de l'observation. Palaeoecol. Africa. 16, $77-88$.

15. Giresse, P. \& Moguedet, G. (1982). Chronoséquences fluvio-marines de l'Holocène de l'estuaire du Kouilou et des colmatages côtiers voisins du Congo. In Les rivages tropicaux - Mangroves d'Afrique et d'Asie. CEGET-CNRS, Travaux et Documents de Géographie tropicale, 39, pp. 21-46.

16. Giresse, P., Malounguila-Nganga, D.M., \& Delibrias, G. (1984). Rythme de la transgression et de la sédimentation holocènes sur les plate-formes sous-marines du sud du Gabon et du Congo. C. R. Académie des Sciences, Paris, 299, II (7), pp.327 - 330.

17. Giresse, P. (1978). Le contrôle climatique de la sédimentation marine et continentale en Afrique centrale atlantique à la fin du Quaternaire. Problème de corrélation. Palaéogéogr. Palaéoclimatol. Palaéoécol., 23, $57-77$.

18. Giresse, P. (1980). Carte sédimentologique du plateau continental du Congo, ORSTOM, notice explicative n ${ }^{\circ} 85$, Editions ORSTOM. 
19. Giresse, P. (1981). Les sédimentogenèses et les morphogenèses quaternaires du plateau et de la côte du Congo en fonction du cadre structural. Bulletin de l'Institut Fondamental d'Afrique Noire, Dakar 43 A $(1-2)$, pp. $43-68$.

20. Giresse, P. \& Ribault, L. (1981). Contribution à l'étude exoscopique des quartz à la reconstitution paléogéographique des derniers épisodes du Quaternaire littorale du Congo. Quaternary Research, 15, pp. 86100.

21. Kjedahl, J. (1883). Neue Methode zur Bestimmung des Stickstoffs in organischen körpern. Z. Anal. Chem. 22 : 366-382.

22. Krumbein, W.C. \& Sloss, L.L. (1963). Stratigraphy and sedimentation. W.H. Freeman et Cie (Edit.), San Francisco, 2e éd., vol. 1, 1963, 660 p.

23. Losson, B. \& Corbonnois, J. (2006). Les modes de sédimentation détritique nouvelle méthode de détermination appliquée à des remplissages endokarstiques, Géologica Belgica, 9/3-4 : pp. 257 265.

24. Makany, L. (1964). La côte atlantique du Congo: cadres géographiques et géologiques, leurs influences sur la répartition de la végétation et sur les possibilités agricoles du territoire. Symposium scientifique de Pekin, pp 891-907.

25. Malouguila-Nganga, D.M., Giresse, P., Boussafir, M., \& Miyouna, T. (2017). Late Holocene swampy forest of Loango Bay (Congo). Sedimentary environments and organic matter deposition. Journal of African Earth Sciences 134 (2017), 419-434.

26. Malounguila-Nganga, D.M. (1983). Les environnements sédimentaires des plateformes du Congo et du Gabon au Quaternaire supérieur d'après les données des vibro-carottages, Thèse 3e cycle, Toulouse III (France). 169 p.

27. Malounguila-Nganga, D.M., Nguie, J., \& Giresse, P. (1990). Les paléoenvironnements quartenaires du colmatage de l'estuaire du Kouilou (Congo), In: Paysages quaternaires de l'Afrique centrale atlantique. Edité par Lanfranchi R., Schwartz D. Collection Didactique. ORSTOM, Paris, pp 89-97.

28. Massengo, A. (1970). Contribution à l'étude stratigraphique, sédimentologique et minéralogique de Série Plio-pleistocène du bassin côtier du Congo-Brazzaville. Thèse de Doctorat 3e cycle, Université. Bordeaux, p. 159.

29. Miyouna, T., Malounguila-Nganag, D.M., Essouli, O. F., NdembéNdembé, A., Moussiessié, J., Kinga-Mouzéo, \& Boudzoumou, F. (2016). Etude paléoenvironnementale des dépôts de la Formation de 
couverture du bassin côtier du Congo. Rev. CAMES, Vol-04, Num01, p. $35-44$

30. Passega, R. (1957). Texture as characterististic of clastic deposition. Ann. Assoc. Petrol. Géol. V 41, pp.1952-1984.

31. Peyrot, B. (1983). Interprétation géomorphologique du littoral et de la façade maritime atlantique de la République Populaire du Congo. Trav. Doc. Géogr. Trop., CEGET, 49 : pp 75-79.

32. Rivière, A. (1977). Méthodes granulométriques. Techniques et interprétations. Masson, Paris, $71 \mathrm{p}$.

33. Samba Kimbata M. J., 1991. Précipitations et bilans de l'eau dans le bassin forestier du Congo et ses marges. Thèse de doctorat d'état, Université Bourgogne. Dijon, 241p.

34. Schwartz, D., Guillet, B. \& Deschamps, R. (1990). Etude de deux flores forestières mi-Holocène (6000-3000 BP) et subactuelle (500 BP) conservés in-situ sur le littoral pontenégrin (Congo). In:

35. Lanfranchi R., Chwartz D. (Eds), Paysages quaternaires de l'Afrique atlantique centrale atlantique. Collection Didactique. ORSTOM, Paris, pp 283-297.

36. Schwartz, D. (1985). Histoire d'un paysage : le Lousséké. Paléoenvironnements quaternaires et podzolisation sur sables Batéké (quarante derniers millénaires, région de Brazzaville, R. P. Congo). Thèse Doctorat ès Sciences Naturelles, Université de Nancy I, 211p.

37. Schwartz, D., Guillet B., \& Dechamp, R. (1990). Etude de deux flores forestières mi-holocène $(6000$ - 3000) et subactuelle (500 B.P.) conserves in-situ sur le littoral Pentenégrin (Congo). In: Paysages quaternaires de l'Afrique Centrale atlantique. Edité par Lanfranchi R., et Schwartz D., (Paris : Coll. Didactique, ORSTOM). Pp. 283 - 297.

38. Sitou, L. \& Tchicaya, J. (1991). L'érosion en cirques dans la région côtière du Congo. Bulletin de la Société Géographique de Liège, 27, 1991, 77-91

39. Stuiver, M. \& Reimer, P.J. (1993). Extend 14C calibration Data base and revised CALIB 3.0 14C age Calibration Program Radiocarbon, 35 (21), $5-230$.

40. Tricart, J. (1965). Principes et méthodes de la géomorphologie. Masson, paris. $496 \mathrm{p}$.

41. Walkey, A. \& Black, A.I. (1934). An examination of Degtjareff method for determining soil organic matter and proposed modification of the chromic acid titration method. Soil Sci ; 37. pp 29-37. 